\title{
Effect of Extreme Weather Events on the Sedimentation of the Bay of Samaná, Dominican Republic (1900-2016)
}

\author{
Delanoy Ramón A. ${ }^{1}$, Díaz-Asencio Misael $^{2} \&$ Méndez-Tejeda, Rafael $^{3}$ \\ ${ }^{1}$ Instituto de Física, Facultad de Ciencias, Universidad Autónoma de Santo Domingo, Ministerio de Educación \\ Superior Ciencia y Tecnología. República Dominicana. Av. Alma Master. Apartado Postal 1355. Santo \\ Domingo. RD. \\ ${ }^{2}$ Division of Oceanology. Ensenada Center for Scientific Research and Higher Education. México. Tijuana No. \\ 3918, Zona Playitas, CP. 22860, Ensenada, B.C. México \\ ${ }^{3}$ Research Laboratory in atmospheric science. University of Puerto Rico at Carolina. Puerto Rico. Po. Box 4800. \\ Carolina Puerto Rico. 00984 \\ Correspondence: Méndez-Tejeda, Rafael, Research Laboratory in atmospheric science. University of \\ Puerto Rico at Carolina. Puerto Rico. Po. Box 4800. Carolina Puerto Rico. 00984. E-mail: \\ rafael.mendez@upr.edu
}

Received: September 5, 2019

Accepted: September 26, 2019

Online Published: September 30, 2019

doi:10.5539/jgg.v11n3p56

URL: http://dx.doi.org/10.5539/jgg.v11n3p56

\begin{abstract}
The Bay of Samaná, formed by tectonism and sedimentation, is delimited to the north by the peninsula of the same name, to the south by the north slope of the Eastern Mountain Range and Los Haitises National Park, to the east by the Atlantic Ocean, and to the west by the ancient Gran Estero, today the Lower Yuna. There follows a process of continuous degradation by the existing tectonic forces and the sediment contributions by the Yuna, Yabón, and La Yeguada rivers to the south as well as by the landslides of the mountainous area of the Samaná Peninsula, during periods of storms and hurricanes. The coastal area of Samaná Bay has altered by $2.17 \mathrm{~km}^{2}$ at the mouth of the Yuna River from 2003-2015. The high turbidity level has affected coral reefs and marine species. The mangroves are lost faster than they are regenerated by the coastline's change. Variations in the elemental compositions of calcium and iron show the terrigenous influence on the dynamics of the bay during Extreme Weather Events (EWP) in the river basins that flow into it. Abrupt changes in the rainfall regime produced an equal change in the estuary sedimentation regime, according to the $210 \mathrm{~Pb}$. In the 2007-2016 period, a column of sediment that reached $38 \mathrm{~cm}$ and a $12 \mathrm{~cm}$ to $8.4 \mathrm{~km}$ column were deposited $4 \mathrm{~km}$ southeast of the municipality of Sánchez and east of the mouth of the Yuna River. The Sedimentary Accumulation Rate is very high, and the content of heavy metals exceeds the threshold values of Table SQuirt.
\end{abstract}

Keywords: Sedimentation, Bay of Semaná, Extreme Event, Dominican Repúblic

\section{Introduction}

The island Hispaniola, located in the arch of the Greater Antilles in the Caribbean region, has its origin in the region's tectonic movements and volcanism. Inside it has several active geological faults (Mann et al., 1984), the most important being the northern one on the northern edge of the Caribbean plate where it collides with the North American plate (Bionini et al., 1984). In the northeastern section, in the study area, the Bay of Samaná, is influenced by a zone of subduction (Figure 1), where tectonic movements are frequent, often producing small earthquakes. In the Bay of Samaná on August 4, 1946, an earthquake of magnitude 8.1 on the Richter scale, the largest earthquake in the Dominican Republic, was recorded by instrumental seismology (Delanoy, 1996). The region moves according to the shifting of the Caribbean plate about $37 \mathrm{~mm}$ per year, which represents a displacement of $63 \mathrm{~cm}$ in 20 years (Bionini et al., 1984) in the region, the Cibao fault, and the North. The Yabón fault, the most important of the eastern mountain range, meets the Cibao fault and the Septentrional fault in Samaná Bay, giving rise to a very complex area from the point of view of structural geology and tectonics (Bird, 1980). Coastal displacements by the tectonic forces that affect the region generate the formation of lagoons, which results in the Atlantic Ocean gaining ground while the bay loses ground (Brink et al., 2004). 


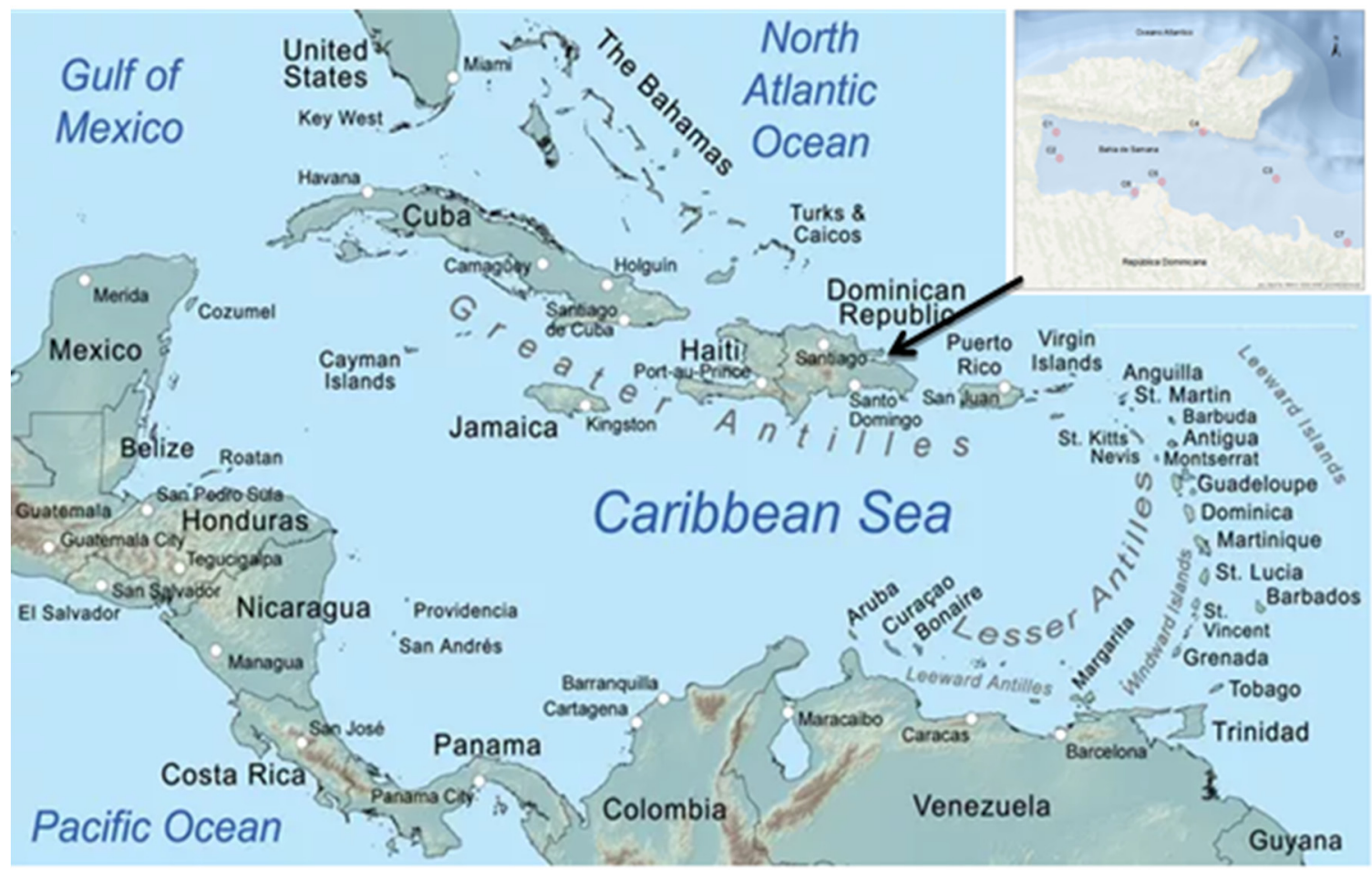

Figure 1. Map of the Caribbean region. The box is the location of the Bay of Samaná in the Dominican Republic Source: https://en.wikipedia.org/wiki/Battle_of_the_Caribbean, https://www.viajarcaribe.es/blog/mapas/

The sediments caused by landslides on the peninsula's escarpments and the drags of the rivers and streams in the northern part of the bay (Eptisa, 2004) during storms and hurricanes are important entities for its degradation (Dolan, 1991). The greatest contributions come from the waters of the Yuna, Yabón, Caño Hondo, and Yeguada rivers that flow south into the bay. The coastal area to the south has been changing due to the contributions of large masses of soils that have been washed away. The use of nuclear and conventional analytical techniques has allowed the reconstruction (Alonso-Hernández et al., 2016) of the degradation process and the factors linked to the sediments in the period from 1900-2016.

The sedimentation rate, physicochemical composition, enrichment, and age are information that we can obtain through these techniques. These parameters are always linked to the displacement of soil masses either by landslides or erosion during heavy rains, which are usually linked to storms or hurricanes and are considered Extreme Weather Events (EWP). Since the 90s, there has been an increase in EWP (NCA, 2018), which affected the Bay of Samaná during the 2004-2012 period, an incidence predominant in the La Niña period (Null, 2017). EWP are frequent in the Dominican Republic because of their location in relation to the routes of tropical waves, hurricanes, and storms that form in the south-central zone of the tropical Atlantic and that are dragged by the trade winds to the northwest, as well as CRCC's (Mendez-Tejeda, 2017). The rivers' sediment contributions during the storms of 2004-2012 were very remarkable (Delanoy et al., (2017). The same happened with the passage of hurricanes Irma and Maria in September 2017.

The Bay of Samaná holds an important economic importance for the Dominican Republic. It is a place to sight humpback whales which, in late winter and early spring, remain in the bay's warm waters to breed. Tourism, fishing, and agriculture are the most important economic sources of livelihood in the towns of Sánchez, Samaná, Sabana de la Mar, and Miches. These are some of the reasons for the importance of studying sedimentation as an element of the ecosystem's (coastal zone and biodiversity) degradation. The study of sediments can provide current and past information since they record the geological and hydrological phenomena of the basin from where they are transported by river waters or wind. The use of nuclear techniques as tools allows reconstructing the past of a basin's activities. Through dating with $210 \mathrm{Pbex}$, a natural radiotracer of the $238 \mathrm{U}$ disintegration chain, it is possible to reconstruct the past (up to 150 years), using sediments as environmental files (Alonso-Hernández, et al., 2016, IAEA 1989, Salamanca et al., 2003, Rodriguez, et al., 2013). This period 
covers industrial development activities and the population growth in the region in the last eight decades (National Bureau of Statistics, Dominican Republic).

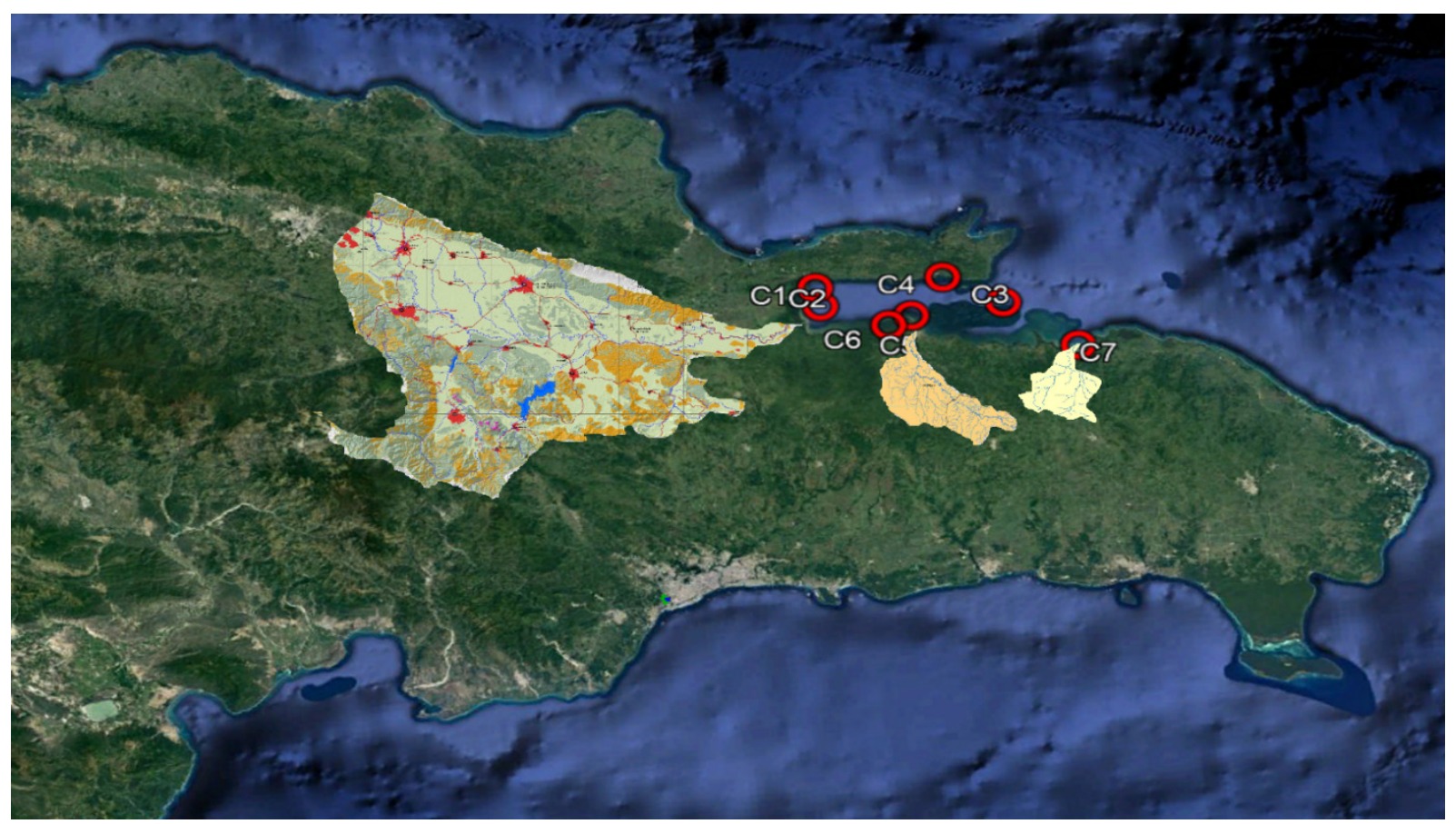

Figure 2. Map of the Dominican Republic. The light-colored areas correspond to the basins of the three main rivers that flow into the Bay of Samaná. Source: Google Earth, Ministry of Environment, Dominican Republic

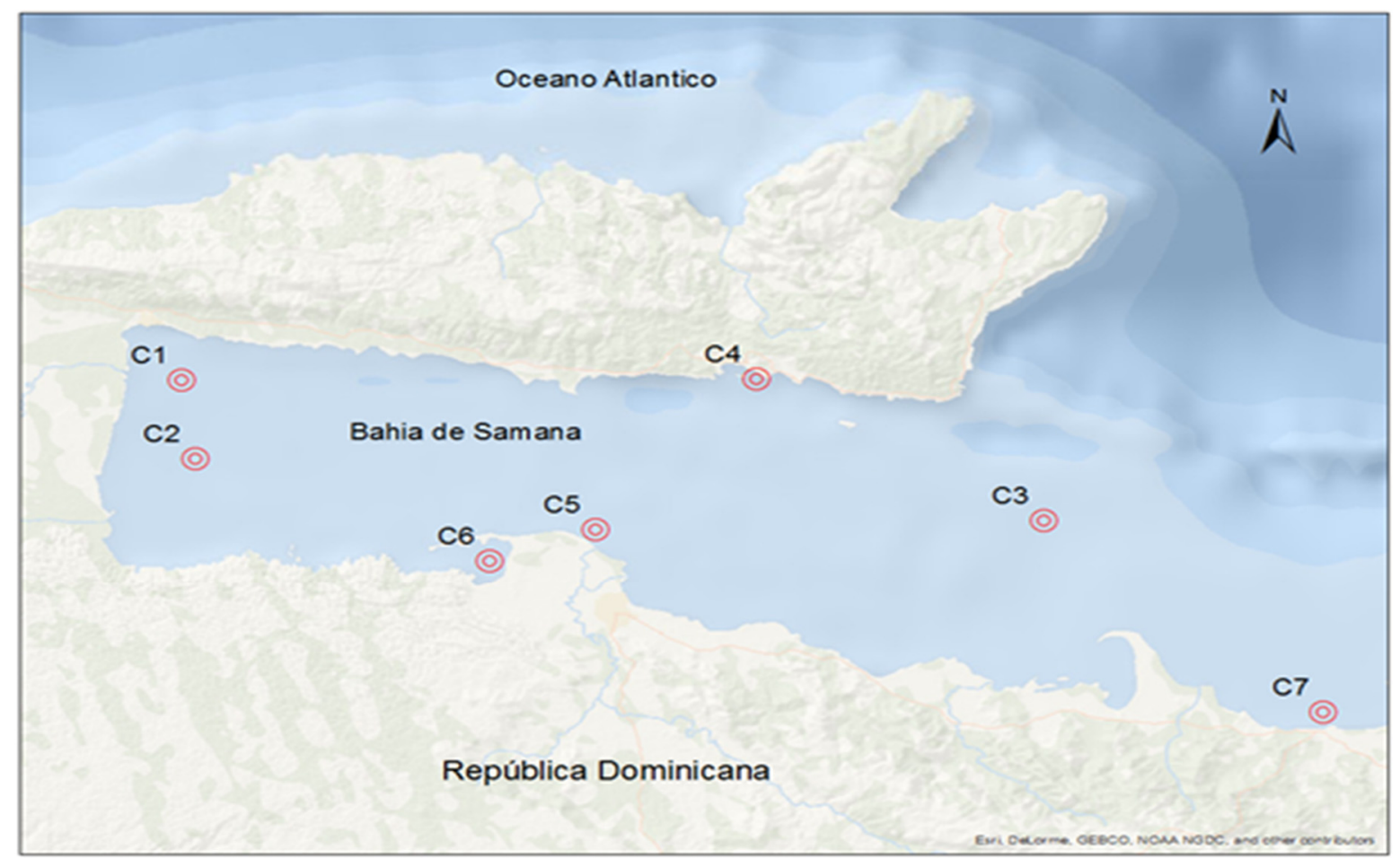

Figure 3. The places where the cores were taken in the area of influence of the rivers that flow into the Samaná Bay

Source: Google Earth 

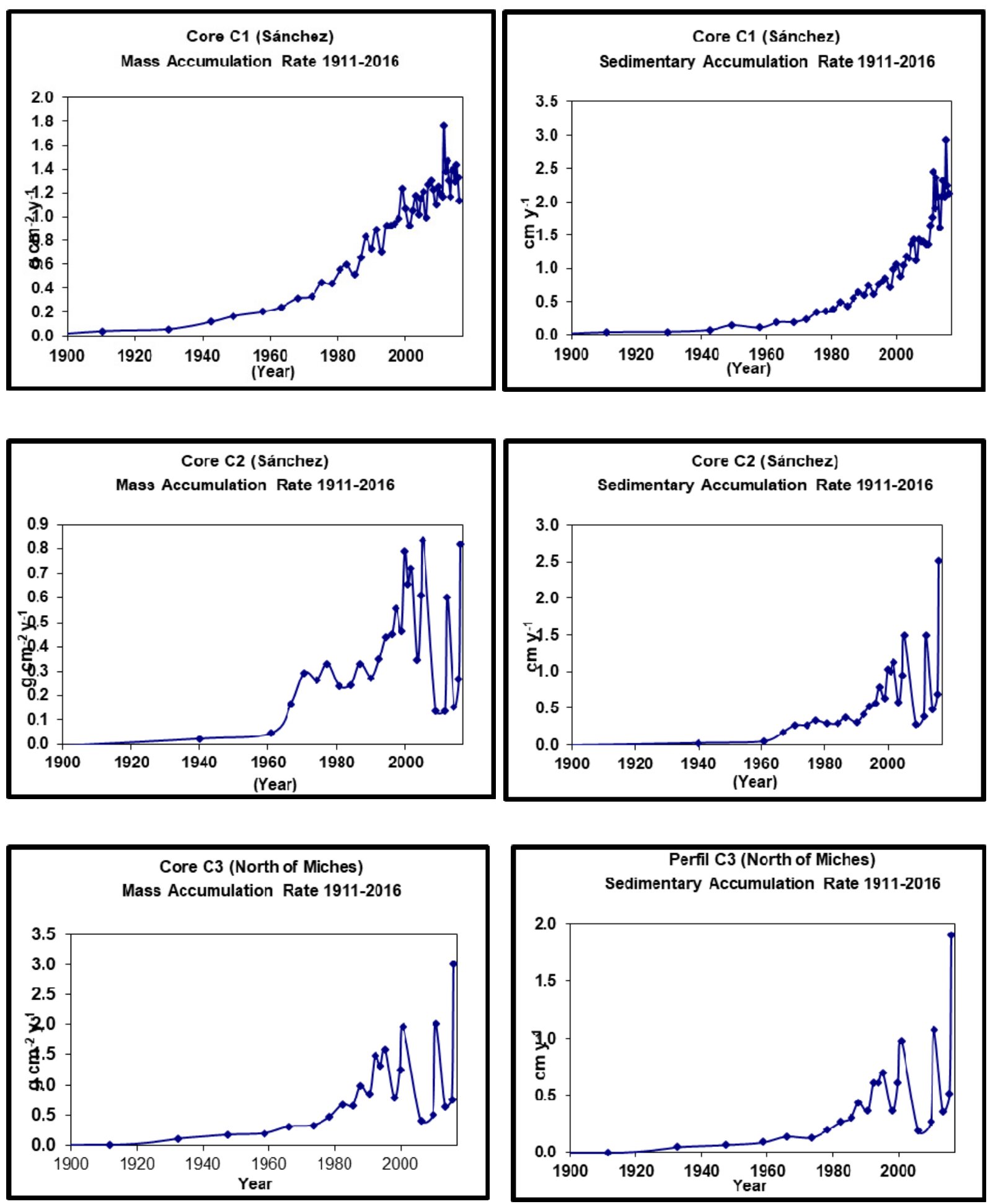

Figure 4. Sedimentary and Mass Accumulation Rate (MAR) according to the lead-210 dating of cores C1, C2, and $\mathrm{C} 3$ 

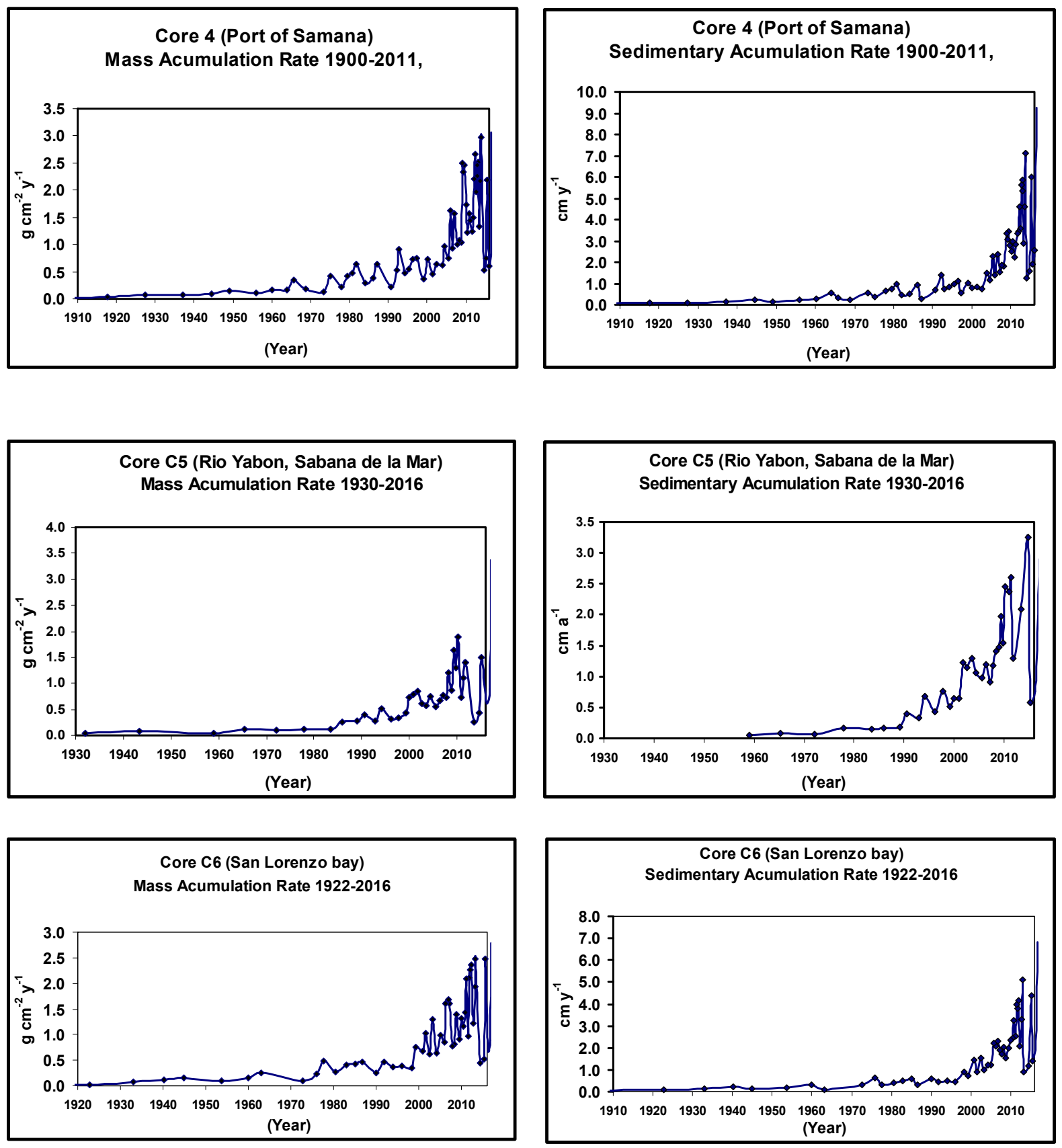

Figure 5. Sedimentary and MAR according to the lead-210 dating of cores C4, C5, and C6

\section{Methods}

\subsection{Study Region}

Core samples were taken at the different mouths of the rivers, the Yuna River and its tributaries the Camú and Jaya rivers cross the central Cibao region, collecting the waters of the Monseñor Noüel, Duarte, Sánchez Ramírez, and La Vega provinces, depositing their waters and eroded soils in the Bay of Samaná. In these provinces are important human settlements dedicated to agriculture, livestock, and mining that contribute to soil erosion and water pollution. Many of the waters used by domestic and hotel services in Samaná and Sánchez, towns located north of the bay, are also discharged to the water sources without treatment and contain metals and solid waste. To the south of the bay are the towns of Sabana de la Mar and Miches, which are related to the sediments that come from the rivers Yabón and La Yeguada, respectively. The main tributaries coming from the north side are the Majagual River and a series of small streams between the towns of Majagual and Sánchez 
(from north to south with little distance between: El Rancho streams, Los Chicharrones, La Jagua, Agua Buena, Las Canoas, La Cabilma, Los Naranjos, and Los Remedios). Several of these tributaries pour their waters into the Yuna near the mouth. Other tributaries are located between the towns of Sánchez and Punta Balandra, from north to south, with little distance between the Punta Gorda, Higüero, Salado, Hondo, Juana Vicenta, Las Flechas, and Los Limones streams and the Santa Capuza, Los Cocos, and the Bushi and Balandra rivers.

\subsection{Yuna-Camu River}

The Yuna river basin covers the south-central, northeast, and part of the north-central Cibao region, a 5,253 $\mathrm{km}^{2}$ area surrounded by a broad-leaved forest that occupies about $44 \%\left(2.311 \mathrm{~km}^{2}\right)$ of the basin. The remaining $56 \%$ is dedicated to four types of intensive agricultural crop uses $\left(848.63 \mathrm{~km}^{2}\right)$, pastures $\left(634.16 \mathrm{~km}^{2}\right)$, rice $(607.27$ $\mathrm{km}^{2}$ ), and cocoa $\left(530.74 \mathrm{~km}^{2}\right)$. The high zones of the Central Mountain Range and the Sierra de Yamasá are covered by $190.28 \mathrm{~km}^{2}$ of coniferous forest (3.62\%) (García-Senz et al., 2007, Hernaiz-Huerta et al., 2004).

\subsection{La Yeguada River (Miches)}

Located in the El Seibo province in the Yuma region, La Yeguada River flows into the municipality of Miches. The area of the basin is $53.7 \mathrm{~km}^{2}$. Cocoa plantations are the main land use coverage in the La Yeguada river basin, comprising an area of $26.5 \mathrm{~km}^{2}(49.3 \%)$. The humid and semihumid broad-leaved forests cover $19.33 \mathrm{~km}^{2}$ (36\%) of the basin. Grass is another land use, with about $7 \mathrm{~km}^{2}$ (12.98\%) of the basin (García-Senz et al., 2007).

\subsection{Yabón River (Sabana de la Mar)}

The Yabón River is located in the Higüamo and Yuma regions and the Hato Mayor and El Seibo provinces. The area of the basin is $370.61 \mathrm{~km}^{2}$. The largest land use is for the cultivation of cocoa, about $107.24 \mathrm{~km}^{2}(28.94 \%)$. Followed by the humid broad-leaved forest with $85.18 \mathrm{~km}^{2}(22.99 \%)$ of the territory, grass occupies the third place with $71.83 \mathrm{~km}^{2}(19.38 \%)$; the land uses with smaller extensions are: dry scrub, coconut, intensive cultures, among others (García-Senz et al., (2007).

\section{Results}

The results of the seven cores are shown in the following tables and graphs using techniques with X-ray fluorescence spectrometry. Tables 1-9 contain some values of the majority and minority elements determined in the nuclei by X-ray fluorescence as well as the sedimentation rate determined by lead-210 dating (Lozano et al., 2011), relating it to the deposition date of the sediments in the mouths of the rivers Yuna, Yabón, La Yeguada, Samaná, and Caño Hondo and one taken inside the Bay of Samaná. This information is related to the sedimentation of the Bay of Samaná due to the dragging of soils during tornadoes, storms, and hurricanes. The increase in iron and calcium in the $\mathrm{C} 1$ core is related to the substitution of organic matter as it degrades (Table 3). The decreases in iron and calcium in sections 20,52, and 70 were due to an increase in mercury concentration.

Potassium values are related to organic matter content and those associated with EWP (Tormenta Olga, 2007, Hurricane George, 1998 and Gert, 1981), according to the date and the mass and sediment accumulation rate (Table 3, Figure 6). In the case of the Gran Estero and Lower Yuna, these sediments have historically been deposited by the river (Dolan, et al., 1998). Iron decreases with depth in the C2 core (Table 4), and calcium increases slightly. The $\mathrm{C} 1$ core showed an increase in calcium and the rate of sedimentation (1998) as a result of Hurricane George (Figure 5). This was taken in the area of influence of the Yuna River, as well as in the C1 core. The $\mathrm{C} 3$ core taken north of Miches, near a galleon loaded with mercury that sunk in the year 1724 during a storm (Fernández-Domingo, 2010), presented higher values of calcium than other nuclei and high mercury values (Table 5, Figure 7).

Table 1. Location, length, and depth of cores taken in the coastal areas of Samaná Bay, 2016-2017, for the study of sedimentation, dated with 210 lead and heavy metals in the last 100 years

\begin{tabular}{cccccc}
\hline Station & Latitude $(\mathrm{N})$ & Longitude $(\mathrm{W})$ & Depth $(\mathrm{cm})$ & Prof. $(\mathrm{m})$ & Site \\
\hline C1 & 19.1926 & 69.5977 & 100 & 28 & Desembocadura río Yuna-Barracote \\
C2 & 19.1460 & 69.5913 & 82 & 25 & Desembocadura río Yuna \\
C3 & 19.1095 & 69.1805 & 46 & 12 & Norte de Miches \\
C4 & 19.1933 & 69.3198 & 58 & 5 & Puerto Samaná \\
C5 & 19.1041 & 69.3977 & 92 & 15 & Río Yabón \\
C6 & 19.0851 & 69.4492 & 52 & 6 & Río Caño Hondo \\
C7 & 18.9958 & 69.0452 & 20 & 6 & Desembocadura Río Yeguada \\
\hline
\end{tabular}


Table 2. Comparison of the certified values of two reference materials BCR 277 and SRM 1944 and the values of these materials obtained with X-ray fluorescence spectrometry, to validate our results in the determination of the major elements and traces

\begin{tabular}{|c|c|c|c|c|c|c|c|}
\hline \multirow[t]{2}{*}{ Element } & \multirow[t]{2}{*}{ Unit } & \multicolumn{3}{|c|}{ Certified value } & \multicolumn{3}{|c|}{$\begin{array}{c}\text { Measured value } \\
\text { XRF }\end{array}$} \\
\hline & & \multicolumn{6}{|c|}{ Concentratión } \\
\hline As & $\mu \mathrm{g} / \mathrm{g}$ & 18.3 & \pm & 1.8 & & & \\
\hline $\mathrm{Cr}$ & $\mu \mathrm{g} / \mathrm{g}$ & 188 & \pm & 14 & 188.0 & \pm & 9.0 \\
\hline $\mathrm{Cu}$ & $\mu \mathrm{g} / \mathrm{g}$ & 63 & \pm & 7.0 & 68.2 & \pm & 1.0 \\
\hline $\mathbf{N i}$ & $\mu \mathrm{g} / \mathrm{g}$ & 130 & \pm & 8.0 & 131.0 & \pm & 4.0 \\
\hline $\mathbf{Z n}$ & $\mu \mathrm{g} / \mathrm{g}$ & 178 & \pm & 20 & 187.0 & \pm & 4.0 \\
\hline \multicolumn{8}{|c|}{ Certified Value SRM 1944} \\
\hline $\mathbf{K}$ & $\%$ & 1.60 & \pm & 0.20 & 1.62 & \pm & 0.04 \\
\hline $\mathbf{C a}$ & $\%$ & 1.00 & \pm & 0.10 & 1.00 & \pm & 0.02 \\
\hline $\mathbf{F e}$ & $\%$ & 3.53 & \pm & 0.16 & 3.54 & \pm & 0.02 \\
\hline $\mathrm{Cr}$ & $\mu \mathrm{g} / \mathrm{g}$ & 266.0 & \pm & 24.0 & 267.40 & \pm & 16.18 \\
\hline $\mathrm{Cu}$ & $\mu \mathrm{g} / \mathrm{g}$ & 380.0 & \pm & 40.0 & 379.13 & \pm & 5.19 \\
\hline Zn & $\mu \mathrm{g} / \mathrm{g}$ & 656.0 & \pm & 75.0 & 656.17 & \pm & 3.35 \\
\hline As & $\mu \mathrm{g} / \mathrm{g}$ & 18.9 & \pm & 2.8 & 18.67 & \pm & 0.21 \\
\hline $\mathrm{Br}$ & $\mu \mathrm{g} / \mathrm{g}$ & 86.0 & \pm & 10.0 & 82.43 & \pm & 3.12 \\
\hline Hg & $\mu \mathrm{g} / \mathrm{g}$ & 3.4 & \pm & 0.5 & 3.30 & \pm & 0.14 \\
\hline $\mathbf{P b}$ & $\mu \mathrm{g} / \mathrm{g}$ & 330.0 & \pm & 48.0 & 329.07 & \pm & 3.92 \\
\hline
\end{tabular}

Table 3. Trace elements, Loss by ignition, $\%$ of Iron, $\%$ of Calcium, Sedimentation rate, Date, and EWP of the sections of core $\mathrm{C} 1$ corresponding to the mouth of the Yuna-Barracote river near the city of Sánchez during the period from 1888-2016. Only sections with mercury higher than $1.0 \mu \mathrm{g} / \mathrm{g}$ were considered

\begin{tabular}{|c|c|c|c|c|c|c|c|c|c|c|c|c|c|c|}
\hline $\begin{array}{l}\text { Proof } \\
(\mathrm{cm})\end{array}$ & $\%$ PPI & $\% \mathrm{Ca}$ & $\% \mathrm{Fe}$ & $\begin{array}{c}\mathrm{Cr} \\
\mu \mathrm{g} / \mathrm{g}\end{array}$ & $\begin{array}{l}\mathrm{Mn} \\
\mu \mathrm{g} / \mathrm{g}\end{array}$ & $\begin{array}{c}\mathrm{Ni} \\
\mu \mathrm{g} / \mathrm{g}\end{array}$ & $\begin{array}{c}\mathrm{Cu} \\
\mu \mathrm{g} / \mathrm{g}\end{array}$ & $\begin{array}{l}\mathrm{Zn} \\
\mu \mathrm{g} / \mathrm{g}\end{array}$ & $\begin{array}{c}\mathrm{Br} \\
\mu \mathrm{g} / \mathrm{g}\end{array}$ & $\begin{array}{c}\mathrm{Hg} \\
\mu \mathrm{g} / \mathrm{g}\end{array}$ & $\begin{array}{c}\mathrm{Pb} \\
\mu \mathrm{g} / \mathrm{g}\end{array}$ & $\begin{array}{l}\text { TAS } \\
\mathrm{cm} / \mathrm{a}\end{array}$ & $\begin{array}{c}\text { Sedimentation } \\
\text { date }\end{array}$ & Storm Date \\
\hline 0 & 17.11 & 2.23 & 6.33 & 253.2 & 651.5 & 95.2 & 70.6 & 105.8 & 820.3 & 1.7 & 24.0 & 2.12 & 2016.1 & \\
\hline 2 & 19.29 & 2.53 & 6.59 & 329.8 & 662.1 & 118.0 & 37.0 & 92.6 & 559.2 & 1.6 & 21.5 & 2.24 & 2015.6 & \\
\hline 4 & 19.07 & 2.41 & 6.55 & 203.5 & 503.1 & 139.5 & 99.4 & 75.8 & 579.3 & 1.0 & 27.2 & 2.93 & 2015.2 & Bertha 2014 \\
\hline 18 & 17.11 & 2.12 & 6.53 & 169.9 & 527.9 & 111.5 & 71.6 & 100.7 & 437.3 & 2.8 & 23.7 & 1.90 & 2011.9 & \\
\hline 20 & 16.85 & 2.22 & 6.09 & 242.5 & 543.9 & 108.1 & 113.9 & 89.3 & 474.6 & 3.9 & 25.6 & 2.45 & 2011.4 & Irene 2011 \\
\hline 22 & 17.41 & 2.51 & 6.59 & 341.9 & 623.2 & 133.1 & 121.3 & 340.6 & 439.3 & 1.9 & 43.1 & 1.76 & 2011.0 & Tomas 2010 \\
\hline 24 & 17.52 & 2.57 & 6.66 & 249.2 & 584.7 & 102.0 & 91.9 & 64.5 & 337.2 & 3.1 & 34.9 & 1.65 & 2010.4 & Earl 2010 \\
\hline 26 & 18.43 & 2.92 & 6.57 & 108.1 & 581.7 & 146.7 & 63.5 & 70.9 & 330.7 & 1.8 & 42.5 & 1.36 & 2009.8 & \\
\hline 30 & 19.34 & 1.82 & 6.43 & 126.9 & 427.6 & 136.1 & 80.0 & 66.8 & 332.7 & 2.5 & 38.0 & 1.40 & 2008.3 & Ike 2008 \\
\hline 36 & 16.95 & 1.31 & 6.04 & 75.8 & 425.7 & 137.6 & 95.5 & 80.7 & 272.1 & 2.6 & 38.4 & 1.12 & 2006.2 & \\
\hline 38 & 18.58 & 2.35 & 6.84 & 305.6 & 551.9 & 120.6 & 98.4 & 158.4 & 362.1 & 4.0 & 29.2 & 1.44 & 2005.3 & Alpha 2005 \\
\hline 40 & 16.61 & 1.61 & 6.26 & 121.5 & 509.6 & 130.4 & 134.9 & 70.4 & 391.2 & 2.8 & 39.9 & 1.37 & 2004.6 & Jeanne 2004 \\
\hline 42 & 17.24 & 2.09 & 6.86 & 230.4 & 753.7 & 118.0 & 52.2 & 110.5 & 311.2 & 5.4 & 45.5 & 1.15 & 2003.9 & \\
\hline 46 & 19.13 & 2.08 & 6.62 & 251.9 & 652.2 & 158.9 & 63.5 & 109.2 & 183.9 & 3.3 & 22.0 & 1.04 & 2002.2 & \\
\hline 52 & 13.64 & 2.21 & 6.95 & 192.8 & 713.6 & 131.2 & 87.1 & 214.2 & 265.6 & 2.9 & 33.8 & 0.98 & 1999.1 & George 1998 \\
\hline 62 & 17.19 & 2.03 & 6.79 & 251.9 & 741.5 & 137.6 & 99.4 & 46.8 & 233.1 & 3.0 & 34.6 & 0.62 & 1993.0 & \\
\hline 64 & 17.10 & 2.60 & 7.08 & 211.6 & 720.5 & 144.5 & 52.2 & 83.2 & 202.8 & 2.7 & 30.3 & 0.75 & 1991.4 & Hugo 1989 \\
\hline 68 & 18.34 & 2.33 & 6.49 & 149.8 & 564.5 & 116.1 & 46.7 & 116.8 & 562.0 & 4.8 & 30.3 & 0.64 & 1988.4 & \\
\hline 70 & 16.91 & 2.42 & 6.68 & 192.8 & 719.4 & 112.3 & 108.7 & 62.1 & 131.3 & 6.0 & 24.5 & 0.56 & 1986.8 & \\
\hline 72 & 17.05 & 3.05 & 7.12 & 183.4 & 733.5 & 164.5 & 98.1 & 87.3 & 131.0 & 1.6 & 34.3 & 0.44 & 1985.0 & \\
\hline 86 & 17.04 & 2.28 & 7.05 & 266.7 & 640.0 & 117.6 & 150.1 & 62.1 & 191.5 & 3.5 & 43.1 & 0.19 & 1963.1 & \\
\hline 90 & 15.31 & 2.75 & 7.35 & 222.3 & 660.6 & 185.0 & 110.4 & 73.2 & 207.3 & 6.1 & 30.8 & 0.15 & 1949.2 & Tsunami 1946 \\
\hline 96 & 13.88 & 2.63 & 7.84 & 194.1 & 725.8 & 202.8 & 89.7 & 74.7 & 121.6 & 1.4 & 40.5 & 0.04 & 1910.5 & \\
\hline
\end{tabular}



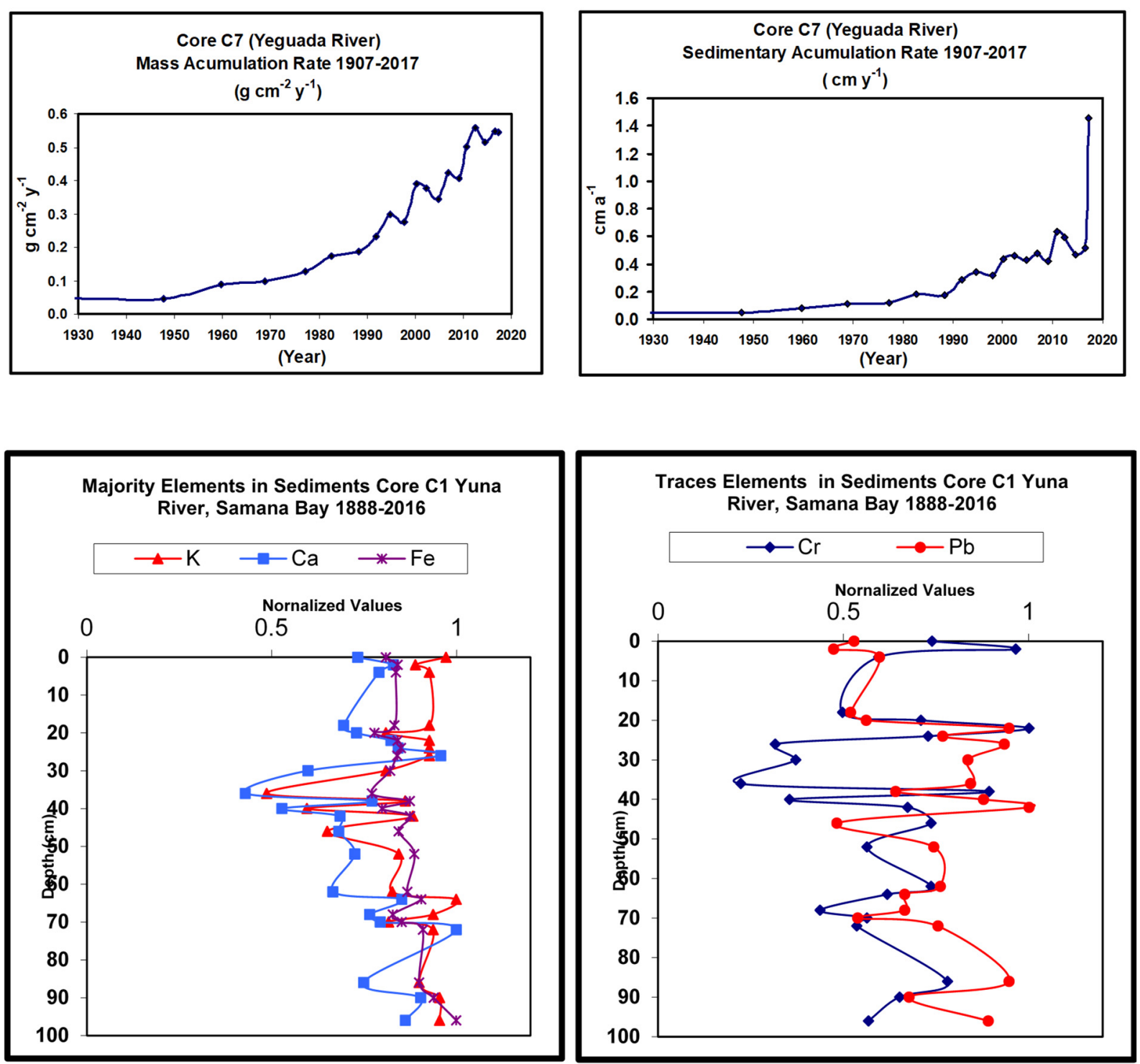

Figure 6. Sedimentary MAR (upper left), MAR (upper right) according to the lead-210 dating of the $\mathrm{C} 7$ core.

Variation with the depth of the majority elements potassium, calcium, and iron (lower left) and minority elements chrome and lead (lower right) found in core $\mathrm{C} 1$ in the Sánchez province 

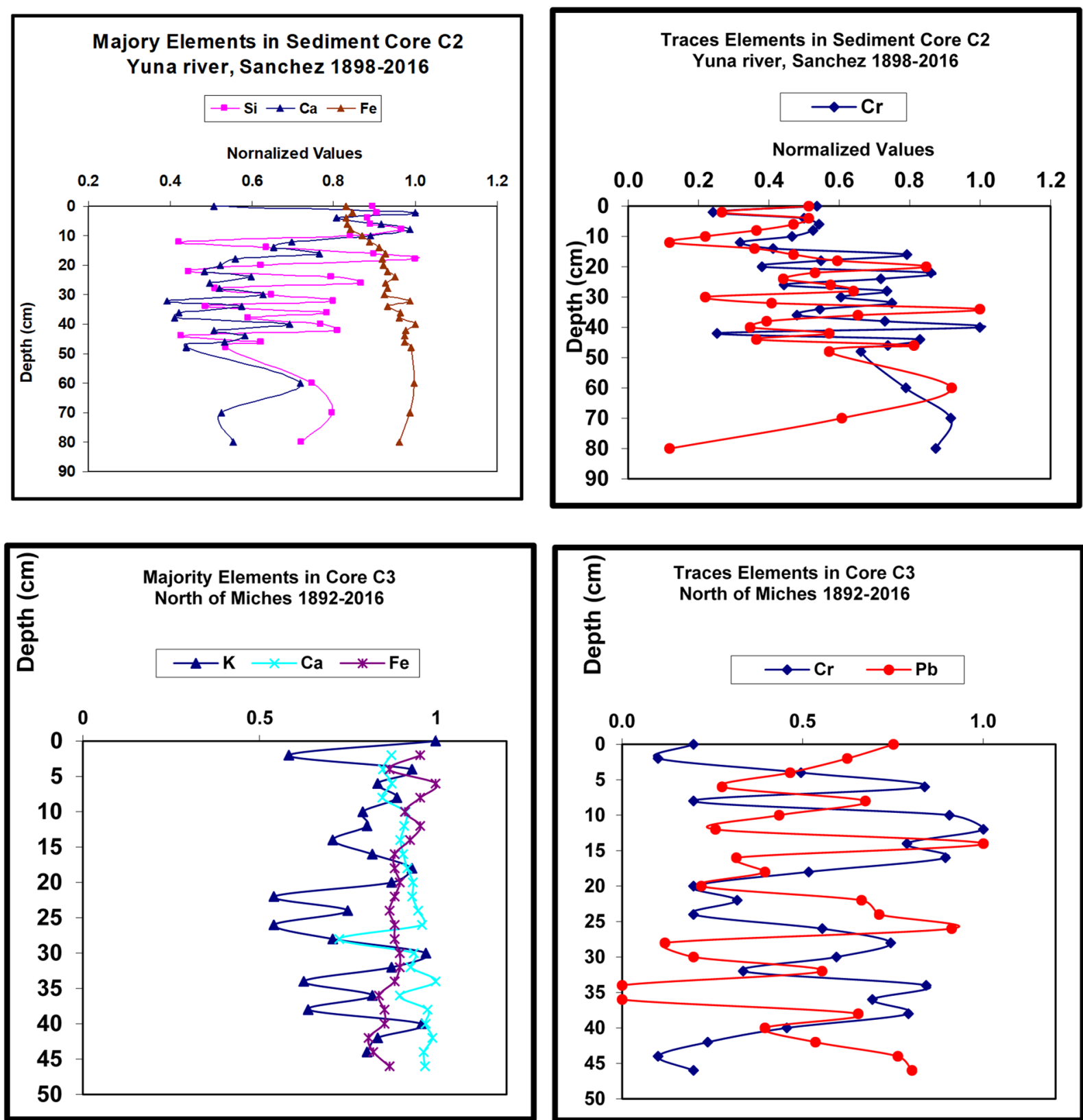

Figure 7. Relationship of the chemical elements with the depth in core C2 (majority: silicon, calcium, and iron; minorities: chromium and lead). Core C3 (majority: potassium, calcium, and iron; minority: chromium and lead) 
Table 4. Trace elements, Loss by ignition, \% of Iron, \% of Calcium, Sedimentation rate, Date, and EWP of the sections of the core $\mathrm{C} 2$ corresponding to the mouth of the Yuna-Barracote river in the south of the Sánchez province during the period from $1898-2016$

\begin{tabular}{|c|c|c|c|c|c|c|c|c|c|c|c|c|c|}
\hline $\begin{array}{l}\text { Proof } \\
(\mathrm{cm})\end{array}$ & $\%$ PPI & $\% \mathrm{Ca}$ & $\% \mathrm{Fe}$ & $\begin{array}{c}\mathrm{Cr} \\
\mu \mathrm{g} / \mathrm{g}\end{array}$ & $\begin{array}{l}\mathrm{Mn} \\
\mu \mathrm{g} / \mathrm{g}\end{array}$ & $\begin{array}{c}\mathrm{Ni} \\
\mu \mathrm{g} / \mathrm{g}\end{array}$ & $\begin{array}{c}\mathrm{Cu} \\
\mu \mathrm{g} / \mathrm{g}\end{array}$ & $\begin{array}{c}\mathrm{Zn} \\
\mu \mathrm{g} / \mathrm{g}\end{array}$ & $\begin{array}{c}\mathrm{Br} \\
\mu \mathrm{g} / \mathrm{g}\end{array}$ & $\begin{array}{c}\mathrm{Pb} \\
\mu \mathrm{g} / \mathrm{g}\end{array}$ & $\begin{array}{l}\text { TAS } \\
\mathrm{cm} / \mathrm{a}\end{array}$ & $\begin{array}{c}\text { Sedimentation } \\
\text { date }\end{array}$ & Storm Date \\
\hline & 14.82 & 1.76 & 5.1 & 190.1 & 412.3 & 86.1 & 44.3 & 119 & 1205.5 & 8.7 & 2.52 & 2016.1 & \\
\hline 2 & 13.22 & 1.54 & 5.19 & 85.3 & 400.5 & 117.2 & 13.9 & 148.1 & 990.3 & 4.5 & 0.68 & 2015.7 & Bertha 2014 \\
\hline 4 & 12.56 & 1.69 & 5.11 & 176.6 & 423.4 & 118 & 47.8 & 216.3 & 877.4 & 8.7 & 0.48 & 2014.2 & Gabrielle 2013 \\
\hline 6 & 12.66 & 1.97 & 5.12 & 191.4 & 497.8 & 116.1 & 9.8 & 203.8 & 876 & 8 & 1.49 & 2012.1 & Isaac 2012 \\
\hline 8 & 13.73 & 2.02 & 5.17 & 186.0 & 466.5 & 114.9 & 34.4 & 182.3 & 837 & 6.2 & 0.38 & 2011.5 & Irene 2011 \\
\hline 10 & 13.65 & 2.18 & 5.34 & 164.5 & 543.1 & 85.4 & 49.8 & 119.4 & 840.9 & 3.7 & 0.28 & 2008.8 & Olga-Noel 2007 \\
\hline 12 & 14.99 & 1.74 & 5.46 & 112.1 & 519.9 & 129.7 & 38 & 132.8 & 630.5 & 6 & 1.49 & 2005.2 & Alpha 2005 \\
\hline 14 & 13.68 & 1.93 & 5.6 & 145.7 & 456.6 & 122.9 & 74.3 & 111.8 & 542.5 & 6.1 & 0.94 & 2004.5 & Jeanne 2004 \\
\hline 16 & 12.79 & 2.09 & 5.7 & 280.1 & 481.7 & 128.2 & 9.7 & 108.3 & 575.6 & 8 & 0.57 & 2003.5 & \\
\hline 18 & 14.35 & 2 & 5.65 & 194.1 & 692.7 & 133.1 & 21.5 & 155.8 & 535.7 & 10.1 & 1.12 & 2001.7 & \\
\hline 20 & 12.5 & 2.02 & 5.66 & 135.0 & 728.1 & 140.3 & 9.9 & 99.9 & 531.8 & 14.4 & 1 & 2000.8 & \\
\hline 22 & 12.89 & 2.13 & 5.72 & 304.3 & 882.6 & 151.3 & 9.6 & 109.1 & 474.4 & 9 & 1.03 & 1999.9 & George 1998 \\
\hline 24 & 11.59 & 3.26 & 5.83 & 254.6 & 921.1 & 166.8 & 21.3 & 108.1 & 386.1 & 7.5 & 0.63 & 1998.9 & \\
\hline 26 & 11.12 & 3.24 & 5.69 & 156.5 & 848.3 & 116.4 & 40.2 & 110.0 & 471 & 9.8 & 0.79 & 1997.3 & Hortensia 1996 \\
\hline 28 & 13 & 1.95 & 5.73 & 260.0 & 1041.7 & 131.2 & 9.5 & 127.5 & 472.4 & 10.9 & 0.57 & 1996.0 & \\
\hline 30 & 12.12 & 2.15 & 5.67 & 214.3 & 901.7 & 108.1 & 9.4 & 121.6 & 386.4 & 3.7 & 0.52 & 1994.3 & \\
\hline 32 & 13.33 & 2.26 & 6.06 & 265.3 & 1026.0 & 141.8 & 55.9 & 115.5 & 472.4 & 6.9 & 0.42 & 1992.3 & \\
\hline 34 & 11.64 & 2.14 & 5.73 & 192.8 & 999.3 & 157 & 36.0 & 78.7 & 418.6 & 17 & 0.31 & 1990.0 & \\
\hline 36 & 15.16 & 2.43 & 5.92 & 169.9 & 1132.1 & 137.3 & 38.3 & 131.3 & 316.8 & 11.1 & 0.38 & 1986.7 & Emely 1987 \\
\hline 38 & 11.27 & 2.25 & 5.91 & 258.6 & 1125.6 & 169.8 & 9.1 & 91.4 & 347.6 & 6.7 & 0.29 & 1984.1 & \\
\hline 40 & 13.34 & 2.53 & 6.14 & 354.0 & 1006.6 & 155.1 & 21.0 & 61.8 & 419.8 & 5.9 & 0.29 & 1980.6 & David Federico 1979 \\
\hline 42 & 13.06 & 2.34 & 6 & 89.3 & 940.6 & 120.2 & 9.5 & 103.0 & 375.1 & 9.7 & 0.33 & 1977.2 & \\
\hline 44 & 13.8 & 2.07 & 5.99 & 293.5 & 994.4 & 130.1 & 16.6 & 86.6 & 312.3 & 6.2 & 0.27 & 1974.2 & \\
\hline 46 & 12.82 & 2.08 & 5.99 & 261.3 & 901.7 & 139.5 & 37.5 & 84.4 & 361.2 & 13.8 & 0.26 & 1970.5 & \\
\hline 48 & 10.75 & 2.29 & 6.08 & 234.4 & 1079.0 & 163 & 55.9 & 105.4 & 250.9 & 9.7 & 0.17 & 1966.7 & \\
\hline 60 & 13.52 & 2.31 & 6.13 & 278.8 & 1146.6 & 155.8 & 35.2 & 91.0 & 379 & 15.6 & 0.05 & 1960.9 & \\
\hline 70 & 13.3 & 2.22 & 6.07 & 324.5 & 1244.2 & 95.6 & 9.6 & 93.4 & 331 & 10.3 & 0.02 & 1939.9 & \\
\hline 80 & 13.51 & 2.36 & 5.91 & 309.7 & 1224.4 & 106.6 & 21.3 & 90.7 & 336 & 9 & 0.01 & 1898.9 & \\
\hline
\end{tabular}


In the Samaná port (Samaná River), the $\mathrm{C} 4$ core was extracted, where calcium and iron decreased with depth (Table 6). Between 22-43 cm, a change in the sedimentation rate was observed (Figure 8). Of terrigenous influence, the Samaná River collects wastewater from Santa Bárbara de Samaná. Changes in the composition of the elements and the rate of sedimentary accumulation correspond to EWP (Figure 8).
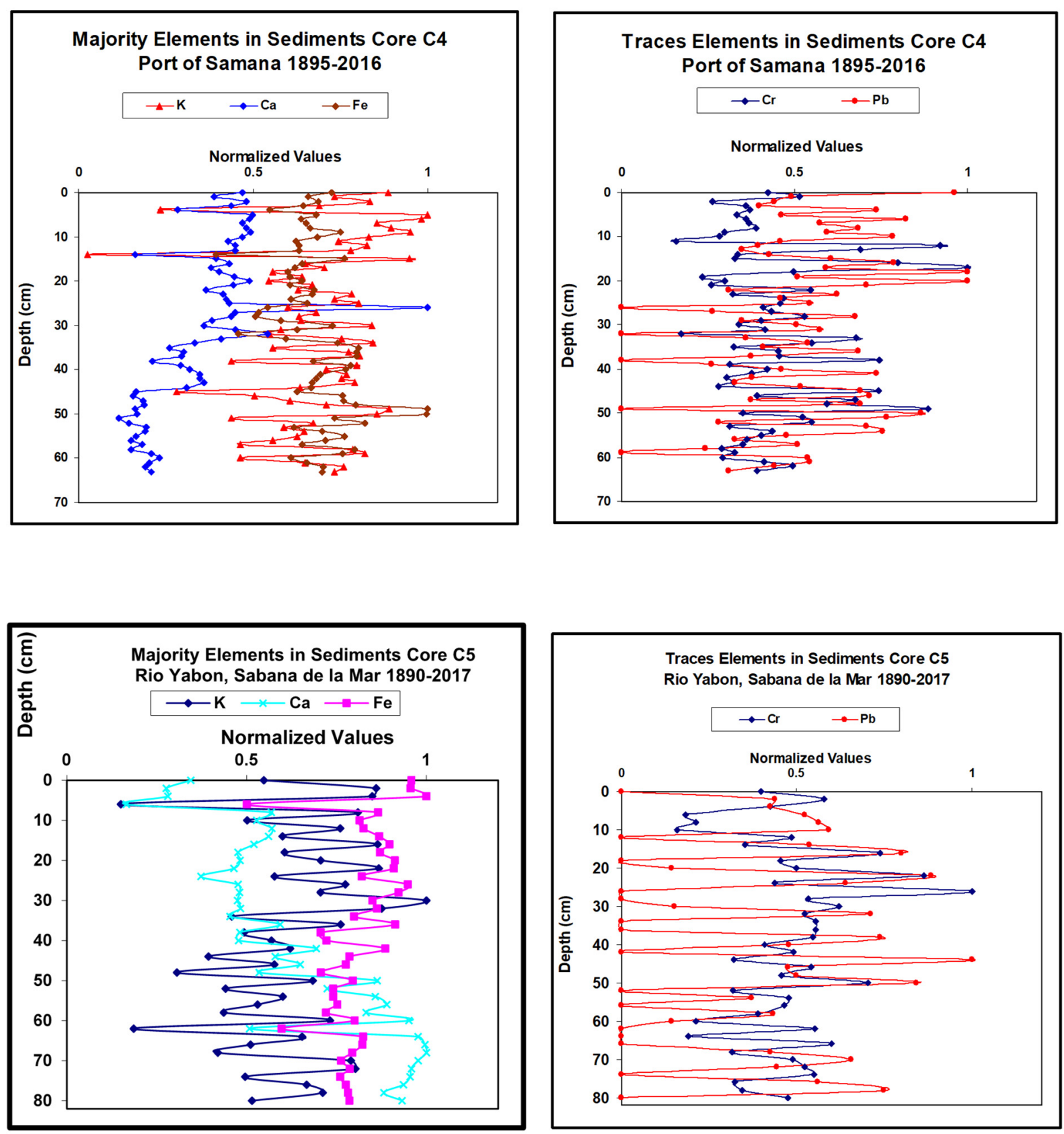

Figure 8. Relationship of the chemical elements with the depth in core $\mathrm{C} 4$ (majorities: potassium, calcium, and iron; minorities: chromium and lead). Core C5 (majority: potassium, calcium, and iron; minority: chromium and lead) 
Table 5. Trace elements, Loss by ignition, $\%$ of Iron, $\%$ of Calcium, Sedimentation rate, Date, and EWP of the sections of the core C3 north of the city of Miches during the period from 1892-2016. We only consider the sections with higher values of lead at $3.9 \mu \mathrm{g} / \mathrm{g}$

\begin{tabular}{|c|c|c|c|c|c|c|c|c|c|c|c|c|c|}
\hline $\begin{array}{l}\text { Proof. } \\
(\mathrm{cm})\end{array}$ & $\%$ PPI & $\% \mathrm{Ca}$ & $\% \mathrm{Fe}$ & $\begin{array}{c}\mathrm{Cr} \\
\mu \mathrm{g} / \mathrm{g}\end{array}$ & $\mathrm{Mn} \mu \mathrm{g} / \mathrm{g}$ & Ni $\mu \mathrm{g} / \mathrm{g}$ & $\mathrm{Cu} \mu \mathrm{g} / \mathrm{g}$ & $\mathrm{Zn} \mu \mathrm{g} / \mathrm{g}$ & $\begin{array}{c}\mathrm{Br} \\
\mu \mathrm{g} / \mathrm{g}\end{array}$ & $\mathrm{Pb} \mu \mathrm{g} / \mathrm{g}$ & $\begin{array}{l}\text { TAS } \\
\mathrm{cm} / \mathrm{a}\end{array}$ & $\begin{array}{c}\text { Sedimentation } \\
\text { date }\end{array}$ & Storm Date \\
\hline 0 & 11.97 & 42.89 & 0.65 & 10.9 & 159.5 & 28.7 & 18.4 & 36.8 & 166.9 & 7.6 & 1.90 & 2016.1 & \\
\hline 2 & 10.81 & 41.75 & 0.59 & 5.5 & 133.4 & 36.9 & 8.8 & 37.5 & 142.3 & 6.3 & 0.51 & 2015.5 & Bertha2014 \\
\hline 4 & 13.55 & 43.00 & 0.68 & 27.3 & 148.3 & 31.3 & 6.7 & 33.2 & 122.2 & 4.7 & 0.35 & 2013.6 & Gabrielle 2013 \\
\hline 8 & 11.91 & 44.89 & 0.62 & 10.9 & 114.8 & 27.7 & 9.6 & 36.1 & 144.0 & 6.8 & 0.27 & 2009.8 & Olga-Noel 2007 \\
\hline 14 & 9.27 & 44.61 & 0.60 & 43.4 & 117.7 & 37.7 & 8.1 & 37.5 & 71.7 & 10.1 & 0.61 & 1999.8 & \\
\hline 18 & 16.97 & 45.97 & 0.61 & 28.4 & 164.5 & 13.7 & 8.2 & 32.2 & 66.2 & 4.0 & 0.69 & 1995.4 & Hortensia 1996 \\
\hline 22 & 22.05 & 46.64 & 0.59 & 17.5 & 65.9 & 9.8 & 9.2 & 38.4 & 58.7 & 6.7 & 0.61 & 1992.3 & \\
\hline 24 & 28.15 & 47.23 & 0.60 & 10.9 & 95.7 & 7.6 & 7.9 & 35.2 & 47.2 & 7.2 & 0.36 & 1990.6 & Hugo 1989 \\
\hline 26 & 9.33 & 35.67 & 0.60 & 30.5 & 52.2 & 39.6 & 8.0 & 32.9 & 64.2 & 9.2 & 0.44 & 1987.9 & Emely 1987 \\
\hline 32 & 13.93 & 49.16 & 0.60 & 18.5 & 61.3 & 46.4 & 7.4 & 33.6 & 65.8 & 5.6 & 0.20 & 1978.5 & David Federico 1979 \\
\hline 34 & 12.10 & 44.08 & 0.57 & 46.4 & 116.4 & 23.1 & 9.6 & 33.5 & 87.7 & 5.6 & 0.13 & 1973.5 & \\
\hline 36 & 16.06 & 47.97 & 0.58 & 38.2 & 278.9 & 10.1 & 6.4 & 28.0 & 67.8 & 6.0 & 0.14 & 1965.8 & \\
\hline 38 & 10.54 & 47.67 & 0.58 & 43.7 & 78.3 & 24.1 & 7.8 & 32.2 & 41.1 & 6.6 & 0.09 & 1958.6 & \\
\hline 40 & 19.87 & 48.63 & 0.55 & 25.1 & 74.6 & 14.5 & 8.4 & 39.0 & 41.1 & 4.0 & 0.07 & 1947.4 & \\
\hline 42 & 7.71 & 47.48 & 0.56 & 13.1 & 116.8 & 7.6 & 9.2 & 34.0 & 56.2 & 5.4 & 0.05 & 1932.4 & \\
\hline 44 & 9.41 & 47.60 & 0.59 & 5.5 & 89.9 & 42.6 & 23.9 & 30.2 & 47.8 & 7.7 & 0.05 & 1911.7 & \\
\hline 46 & 16.62 & 47.29 & 0.54 & 10.9 & 105.2 & 19.5 & 8.5 & 34.2 & 57.8 & 8.1 & 0.05 & 1892.5 & \\
\hline
\end{tabular}

Table 6. Trace elements, Loss by ignition, \% of Iron, \% of Calcium, Sedimentation rate, Date, and EWP of the sections of the $\mathrm{C} 4$ core corresponding to the mouth of the Samaná River in the port of Samaná City during the period from 1895-2016. We only consider the sections with higher values of lead at $20 \mu \mathrm{g} / \mathrm{g}$

\begin{tabular}{|c|c|c|c|c|c|c|c|c|c|c|c|c|c|}
\hline $\begin{array}{l}\text { Proof } \\
(\mathrm{cm})\end{array}$ & $\%$ PPI & $\% \mathrm{Ca}$ & $\% \mathrm{Fe}$ & $\begin{array}{c}\mathrm{Cr} \\
\mu \mathrm{g} / \mathrm{g}\end{array}$ & $\begin{array}{c}\mathrm{Mn} \\
\mu \mathrm{g} / \mathrm{g}\end{array}$ & $\begin{array}{c}\mathrm{Ni} \\
\mu \mathrm{g} / \mathrm{g}\end{array}$ & $\begin{array}{c}\mathrm{Zn} \\
\mu \mathrm{g} / \mathrm{g}\end{array}$ & $\begin{array}{c}\mathrm{Br} \\
\mu \mathrm{g} / \mathrm{g}\end{array}$ & $\begin{array}{r}\mathrm{Hg} \\
\mu \mathrm{g} / \mathrm{g}\end{array}$ & $\begin{array}{c}\mathrm{Pb} \\
\mu \mathrm{g} / \mathrm{g}\end{array}$ & $\begin{array}{l}\text { TAS } \\
\mathrm{cm} / \mathrm{s}\end{array}$ & $\begin{array}{c}\text { Sedimentation } \\
\text { date }\end{array}$ & Storm date \\
\hline 0 & 16.92 & 3.43 & 3.35 & 232.6 & 482.6 & 51.7 & 44.5 & 137.1 & & 48.6 & 9.26 & 2016.5 & \\
\hline 2 & 16.56 & 3.52 & 3.18 & 144.7 & 555.9 & 103.0 & 61.7 & 114.5 & & 22.3 & 1.89 & 2016.0 & \\
\hline 4 & 18.31 & 2.07 & 2.53 & 203.9 & 396.6 & 159.8 & 46.8 & 83.6 & & 37.1 & 1.6 & 2015.3 & Bertha 2014 \\
\hline 6 & 16.75 & 3.58 & 2.95 & 197.9 & 428 & 114.1 & 40.8 & 89.8 & & 41.5 & 7.12 & 2013.9 & Gabrielle 2013 \\
\hline 8 & 21.02 & 3.53 & 3.07 & 213.2 & 455 & 142.2 & 40.2 & 82.4 & & 34.5 & 2.9 & 2013.6 & \\
\hline 10 & 15.89 & 3.44 & 3.16 & 156.5 & 469 & 211.0 & 60.1 & 85.9 & & 39.5 & 5.37 & 2013.0 & Dorian 2012 \\
\hline 14 & 16.62 & 1.2 & 1.82 & 184.3 & 142.1 & 110.5 & 27.9 & 64.4 & & 21.5 & 3.46 & 2012.2 & Maria 2011 \\
\hline 15 & 13.93 & 2.88 & 3.52 & 180.6 & 505.7 & 66.0 & 65.4 & 74.8 & & 30.5 & 3.35 & 2011.9 & Irene 2011 \\
\hline 16 & 13.87 & 3.16 & 2.96 & 439.4 & 432.3 & 183.6 & 37.9 & 69.1 & & 39.7 & 2.85 & 2011.6 & \\
\hline 18 & 15.36 & 2.94 & 2.78 & 274.1 & 414.7 & 233 & 41.7 & 62.3 & & 50.4 & 2.99 & 2010.8 & Earl 2010 \\
\hline 21 & 13.4 & 3.25 & 2.8 & 142.4 & 443 & 133.7 & 37.4 & 59.4 & & 35.7 & 3.43 & 2009.7 & Henri 2009 \\
\hline 24 & 12.92 & 3.1 & 2.81 & 257.9 & 376.2 & 189.9 & 39.5 & 59.8 & & 23.1 & 1.83 & 2008.8 & \\
\hline 28 & 9.9 & 3.21 & 2.34 & 291.7 & 406 & 193.8 & 29.8 & 48.3 & 4.2 & 34.1 & 1.4 & 2006.6 & \\
\hline 30 & 12.4 & 2.64 & 3.36 & 186.9 & 492 & 156.6 & 34.5 & 57.1 & & 25.5 & 1.14 & 2005.5 & \\
\hline 31 & 12.03 & 3.29 & 2.89 & 228.1 & 447.9 & 174.1 & 35.7 & 46 & & 28.9 & 1.48 & 2004.6 & Jeanne 2004 \\
\hline 34 & 13.4 & 2.44 & 3.43 & 302.4 & 499.1 & 120.8 & 56.3 & 49.3 & & 27.2 & 0.81 & 2001.3 & \\
\hline 36 & 13.51 & 2.21 & 3.68 & 248.9 & 507.4 & 87.8 & 54.1 & 48 & & 34.5 & 0.56 & 1999.1 & \\
\hline 40 & 13.19 & 2.34 & 3.54 & 232.2 & 454.1 & 160.6 & 50.4 & 46.8 & & 23.3 & 0.72 & 1994.3 & \\
\hline 41 & 13.2 & 2.54 & 3.2 & 207.9 & 541.7 & 100.5 & 56.3 & 51.6 & & 37.1 & 1.42 & 1992.9 & H. Klaus 1990 \\
\hline 44 & 10.95 & 2.28 & 3.07 & 153.8 & 463.2 & 119.9 & 57.2 & 40.2 & & 26.1 & 0.94 & 1987.1 & Emily 1987 \\
\hline 46 & 15.73 & 1.15 & 3.49 & 215.2 & 404.5 & 223.4 & 47.6 & 44.2 & 2.9 & 36.1 & 0.45 & 1984.1 & \\
\hline 48 & 16.02 & 1.38 & 3.67 & 325.9 & 414.5 & 199.7 & 40.4 & 50.7 & & 34.8 & 0.74 & 1980.9 & David Federico 1979 \\
\hline 50 & 14.09 & 1.23 & 4.61 & 193.2 & 285.2 & 154.4 & 53.6 & 43.4 & & 43.7 & 0.36 & 1978.0 & \\
\hline 51 & 14.93 & 0.86 & 3.38 & 287.8 & 228.6 & 145.4 & 28.3 & 42 & & 38.6 & 0.58 & 1975.2 & Eloise 1975 \\
\hline 54 & 15.19 & 1.4 & 3.23 & 239.7 & 440 & 163.3 & 31.2 & 52.9 & & 38.0 & 0.56 & 1965.8 & Faith 1966 \\
\hline 55 & 13.68 & 1.2 & 3.51 & 221.5 & 329.8 & 193.4 & 29 & 43.5 & & 24.1 & 0.26 & 1964.0 & \\
\hline 57 & 14.98 & 1.34 & 2.95 & 193.8 & 221 & 215.9 & 31.3 & 49 & & 25.7 & 0.14 & 1955.9 & \\
\hline 60 & 12.09 & 1.7 & 2.81 & 160.1 & 340.4 & 183.6 & 38.5 & 41.9 & & 27.2 & 0.1 & 1937.2 & \\
\hline 61 & 13.78 & 1.49 & 3.03 & 227.4 & 263.7 & 107.5 & 36.8 & 52.6 & & 27.5 & 0.1 & 1927.3 & \\
\hline 62 & 14.98 & 1.4 & 3.24 & 272.5 & 388.6 & 145.8 & 51.3 & 48.5 & & 22.3 & 0.05 & 1917.6 & \\
\hline
\end{tabular}


Table 7. Trace elements, Loss by ignition, $\%$ of Iron, $\%$ of Calcium, Sedimentation rate, Date, and EWP of the sections of the C5 core corresponding to the Yabón river mouth during the period from 1890-2017. We only consider the sections with higher values of lead at $10 \mu \mathrm{g} / \mathrm{g}$

\begin{tabular}{|c|c|c|c|c|c|c|c|c|c|c|c|c|}
\hline $\begin{array}{l}\text { Proof } \\
(\mathrm{cm})\end{array}$ & $\%$ PPI & $\% \mathrm{Ca}$ & $\% \mathrm{Fe}$ & $\begin{array}{c}\mathrm{Cr} \\
\mu \mathrm{g} / \mathrm{g}\end{array}$ & $\mathrm{Mn} \mu \mathrm{g} / \mathrm{g}$ & $\begin{array}{c}\mathrm{Ni} \\
\mu \mathrm{g} / \mathrm{g}\end{array}$ & $\mathrm{Zn} \mu \mathrm{g} / \mathrm{g}$ & $\mathrm{Br} \mu \mathrm{g} / \mathrm{g}$ & $\mathrm{Pb} \mu \mathrm{g} / \mathrm{g}$ & $\begin{array}{l}\text { TAS } \\
\mathrm{cm} / \mathrm{a}\end{array}$ & $\begin{array}{c}\text { Sedimentation } \\
\text { date }\end{array}$ & Storm date \\
\hline 2 & 16.44 & 1.03 & 4.94 & 158.7 & 725.1 & 93.5 & 22.4 & 86.0 & 11.8 & 1.27 & 2017.0 & \\
\hline 4 & 14.56 & 1.05 & 5.16 & 116.4 & 716.7 & 32.3 & 28.1 & 82.1 & 11.5 & 0.92 & 2016.2 & \\
\hline 6 & 19.98 & 0.62 & 2.59 & 49.9 & 346.7 & 51.8 & 8.3 & 88.9 & 14.1 & 2.90 & 2015.1 & Bertha 2014 \\
\hline 8 & 15.37 & 2.12 & 4.47 & 57.9 & 620.7 & 123.6 & 27.9 & 96.8 & 15.2 & 0.76 & 2014.8 & Gabrielle 2013 \\
\hline 10 & 20.78 & 1.96 & 4.20 & 44.0 & 678.9 & 74.5 & 11.8 & 97.5 & 16.0 & 0.58 & 2013.5 & \\
\hline 14 & 17.92 & 2.09 & 4.49 & 96.9 & 660.8 & 17.3 & 24.5 & 87.2 & 14.5 & 2.08 & 2011.4 & Irene 2011 \\
\hline 16 & 16.85 & 1.94 & 4.63 & 202.3 & 679.4 & 87.5 & 34.2 & 92.2 & 21.6 & 1.30 & 2010.9 & Earl 2010 \\
\hline 22 & 18.26 & 1.74 & 4.70 & 236.9 & 530.9 & 62.4 & 27.8 & 81.3 & 23.9 & 2.46 & 2009.4 & \\
\hline 24 & 20.24 & 1.39 & 4.24 & 119.8 & 547.4 & & 29.0 & 76.9 & 17.3 & 1.55 & 2009.0 & \\
\hline 32 & 17.10 & 1.80 & 4.45 & 143.7 & 637.1 & 87.8 & 22.4 & 73.4 & 19.2 & 1.18 & 2006.4 & Rita 2005 \\
\hline 38 & 19.89 & 1.79 & 3.64 & 150.3 & 578.9 & 72.4 & 12.6 & 76.4 & 19.9 & 0.98 & 2003.6 & \\
\hline 40 & 18.40 & 1.78 & 3.73 & 112.3 & 489.7 & 35.4 & 11.8 & 72.2 & 12.9 & 1.07 & 2002.6 & \\
\hline 44 & 18.33 & 2.16 & 4.06 & 87.8 & 620.0 & 60.4 & 21.4 & 89.3 & 27.0 & 1.14 & 2000.9 & \\
\hline 46 & 19.06 & 2.42 & 4.00 & 148.2 & 545.9 & 65.0 & 27.9 & 80.1 & 12.8 & 1.23 & 2000.0 & \\
\hline 48 & 19.23 & 2.00 & 3.65 & 125.1 & 530.6 & 93.0 & 25.6 & 79.9 & 13.5 & 0.65 & 1999.2 & George 1998 \\
\hline 50 & 18.73 & 3.22 & 4.11 & 192.7 & 581.8 & 75.2 & 24.2 & 82.0 & 22.7 & 0.64 & 1997.7 & \\
\hline 54 & 22.99 & 3.20 & 3.83 & 131.1 & 625.9 & 53.7 & 21.6 & 85.0 & 10.0 & 0.76 & 1994.2 & \\
\hline 58 & 18.54 & 3.11 & 3.72 & 107.2 & & 119.0 & 25.4 & 77.8 & 11.7 & 0.68 & 1990.6 & Klaus 1990 \\
\hline 68 & 16.15 & 3.73 & 4.10 & 87.0 & 523.6 & & 12.1 & 65.3 & 11.5 & 0.15 & 1972.1 & \\
\hline 70 & 15.70 & 3.64 & 3.94 & 134.0 & 578.1 & 111.0 & 10.5 & 57.0 & 17.7 & 0.16 & 1965.3 & \\
\hline 72 & 17.55 & 3.57 & 4.06 & 143.3 & 666.1 & 77.9 & 22.9 & 58.3 & 12.0 & 0.06 & 1959.0 & \\
\hline 76 & 17.86 & 3.49 & 4.00 & 89.0 & 530.9 & & 24.9 & 55.3 & 15.1 & 0.05 & 1932.0 & \\
\hline 78 & 17.25 & 3.29 & 4.04 & 95.0 & 587.9 & 67.5 & 24.8 & 57.4 & 20.2 & 0.05 & 1911.0 & \\
\hline
\end{tabular}

Table 8. Trace elements, Loss by ignition, $\%$ of Iron, $\%$ of Calcium, Sedimentation rate, Date, and EWP of the sections of the core C6 corresponding to the Caño Hondo river mouth, San Lorenzo Bay, during the period from 1922-2017. We only consider the sections with higher lead values at $5.0 \mu \mathrm{g} / \mathrm{g}$. In section 47 the increase in TAS could correspond to an older sediment mixture during earthquakes in the area in 1946

\begin{tabular}{|c|c|c|c|c|c|c|c|c|c|c|c|c|}
\hline $\begin{array}{l}\text { Proof } \\
(\mathrm{cm})\end{array}$ & $\%$ PPI & $\% \mathrm{Ca}$ & $\% \mathrm{Fe}$ & $\begin{array}{r}\mathrm{Cr} \\
\mu \mathrm{g} / \mathrm{g} \\
\end{array}$ & $\begin{array}{r}\mathrm{Mn} \\
\mu \mathrm{g} / \mathrm{g}\end{array}$ & $\begin{array}{r}\mathrm{Ni} \\
\mu \mathrm{g} / \mathrm{g}\end{array}$ & $\begin{array}{c}\mathrm{Zn} \\
\mu \mathrm{g} / \mathrm{g}\end{array}$ & $\begin{array}{r}\mathrm{Br} \\
\mu \mathrm{g} / \mathrm{g} \\
\end{array}$ & $\begin{array}{r}\mathrm{Pb} \\
\mu \mathrm{g} / \mathrm{g}\end{array}$ & $\begin{array}{r}\text { TAS } \\
\mathrm{cm} / \mathrm{a} \\
\end{array}$ & $\begin{array}{c}\text { Sedimentation } \\
\text { date }\end{array}$ & Storm daate \\
\hline 0 & 14.42 & 1.22 & 4.43 & 194 & 562.8 & 103.7 & 11.4 & 157.9 & 13.3 & 6.83 & 2016.9 & \\
\hline 2 & 13.9 & 1.21 & 4.44 & 238.8 & 561.3 & 65.2 & 18 & 107.1 & 14.2 & 1.38 & 2016.2 & \\
\hline 6 & 13.54 & 1.34 & 4.51 & 180.8 & 462.8 & 84.7 & 16.8 & 93.5 & 14.6 & 5.09 & 2013.3 & Gabrielle 2013 \\
\hline 10 & 13.38 & 1.26 & 4.62 & 247.8 & 453.8 & 57.1 & 10.7 & 90 & 16.5 & 3.79 & 2012.1 & Dorian 2012 \\
\hline 11 & 14.51 & 1.41 & 4.63 & 249.8 & 419.6 & 14.3 & 15.4 & 86.3 & 22.3 & 3.96 & 2011.8 & Maria 2011 \\
\hline 13 & 14.63 & 1.25 & 4.71 & 222.5 & 480.8 & 52.6 & 15.6 & 93.8 & 15.1 & 3.23 & 2011.2 & Earl 2010 \\
\hline 14 & 14.47 & 1.29 & 4.8 & 282.7 & 351.3 & 6.5 & 17.9 & 94.7 & 5.1 & 2.42 & 2010.9 & \\
\hline 19 & 12.92 & 1.16 & 4.56 & 284.7 & 459.6 & 29.9 & 21.3 & 85.5 & 32.3 & 1.7 & 2008.4 & Noel Olga 2007 \\
\hline 20 & 13.79 & 1.3 & 4.64 & 158.7 & 445.8 & 17.9 & 9.6 & 86.6 & 18.1 & 1.88 & 2007.8 & \\
\hline 31 & 10.84 & 1.29 & 4.64 & 351.3 & 543.6 & & 4.6 & 66.5 & 20.9 & 0.91 & 1999.4 & George 1998 \\
\hline 32 & 10.74 & 1.14 & 4.65 & 250.8 & 491.4 & 26.2 & 15.6 & 59.3 & 16.8 & 0.44 & 1998.3 & \\
\hline 34 & 11.09 & 1.21 & 4.88 & 228.7 & 510.2 & 4.4 & 12.3 & 58.5 & 29.9 & 0.46 & 1993.9 & \\
\hline 35 & 10.37 & 1.23 & 4.66 & 208.1 & 536.7 & 27.2 & & 57.8 & 11.8 & 0.57 & 1991.8 & Klaus 1990 \\
\hline 36 & 10.62 & 1.29 & 4.78 & 321.6 & 383.1 & 65.7 & 14.2 & 51.6 & 9.2 & 0.3 & 1990.0 & \\
\hline 37 & 10.87 & 1.19 & 4.79 & 138.9 & 592.3 & 78.3 & 10 & 55.7 & 14.2 & 0.58 & 1986.7 & \\
\hline 39 & 11.24 & 1.2 & 4.85 & 296.6 & 431.8 & & 17.3 & 66.4 & 9.4 & 0.42 & 1982.9 & Gert 1981 \\
\hline 44 & 11.42 & 1.23 & 4.47 & 252.6 & 448.7 & 37.8 & 7.1 & 61.1 & 16.6 & 0.32 & 1963.1 & H. Flora 1963 \\
\hline 45 & 11.15 & 1.13 & 4.83 & 364.7 & 517.8 & 64.5 & 8.3 & 58.4 & 14.2 & 0.16 & 1959.9 & \\
\hline 46 & 11.75 & 1.26 & 4.98 & 205.3 & 424.6 & 21.5 & 5.9 & 64.2 & 5.5 & 0.11 & 1953.6 & \\
\hline 47 & 11.01 & 1.19 & 4.65 & 259.8 & 413.6 & 40.3 & 4.6 & 57.2 & 13.4 & 0.21 & 1944.9 & Tsunami 1946 \\
\hline
\end{tabular}


Table 9. Trace elements, Loss by ignition, $\%$ of Iron, $\%$ of Calcium, Sedimentation rate, Date, and EWP of the sections of the $\mathrm{C} 7$ core corresponding to the Yeguada river mouth in the city of Miches during the period from 1947-2017. We only consider the sections with higher values of lead at $5.0 \mu \mathrm{g} / \mathrm{g}$

\begin{tabular}{|c|c|c|c|c|c|c|c|c|c|c|c|c|}
\hline $\begin{array}{l}\text { Proof } \\
(\mathrm{cm})\end{array}$ & $\%$ PPI & $\% \mathrm{Ca}$ & $\% \mathrm{Fe}$ & $\begin{array}{c}\mathrm{Cr} \\
\mu \mathrm{g} / \mathrm{g}\end{array}$ & $\mathrm{Mn} \mu \mathrm{g} / \mathrm{g}$ & $\begin{array}{c}\mathrm{Ni} \\
\mu \mathrm{g} / \mathrm{g}\end{array}$ & $\begin{array}{c}\mathrm{Br} \\
\mu \mathrm{g} / \mathrm{g}\end{array}$ & $\begin{array}{c}\mathrm{Hg} \\
\mu \mathrm{g} / \mathrm{g}\end{array}$ & $\begin{array}{c}\mathrm{Pb} \\
\mu \mathrm{g} / \mathrm{g}\end{array}$ & $\begin{array}{l}\text { TAS } \\
\mathrm{cm} / \mathrm{a}\end{array}$ & $\begin{array}{c}\text { Sedimentation } \\
\text { date }\end{array}$ & Storm date \\
\hline 0 & 11.08 & 16.36 & 1.34 & 75.9 & 411.8 & 101.1 & 23.6 & 2.7 & & 1.46 & 2017.2 & \\
\hline 1 & 11.51 & 18.47 & 0.89 & 52.2 & 325.3 & 125.7 & 19.6 & & & 0.52 & 2016.5 & Bertha 2014 \\
\hline 2 & 9.52 & 8.74 & 2.35 & 68.3 & 477.4 & 100.9 & 40.4 & & 19.4 & 0.47 & 2014.6 & \\
\hline 3 & 11.08 & 10.80 & 1.93 & 137.6 & 435.2 & 73.1 & 32.9 & 4.4 & 20.6 & 0.59 & 2012.4 & \\
\hline 4 & 7.99 & 9.51 & 2.30 & 139.0 & 438.8 & 98.5 & 31.8 & & 26.4 & 0.63 & 2010.8 & \\
\hline 5 & 7.90 & 10.66 & 1.65 & 77.9 & 542.6 & 126.2 & 39.9 & & 18.8 & 0.42 & 2009.2 & \\
\hline 6 & 7.65 & 18.16 & 0.95 & 79.4 & 320.2 & 37.3 & 18.5 & & 22.3 & 0.48 & 2006.8 & Jeanne 2004 \\
\hline 7 & 7.70 & 17.40 & 0.86 & 51.9 & 364.4 & 70.7 & 18.8 & & & 0.43 & 2004.7 & \\
\hline 8 & 9.93 & 17.49 & 0.98 & 50.5 & 336.2 & 116.5 & 21.4 & & 6.2 & 0.46 & 2002.4 & Debby 2000 \\
\hline 9 & 11.66 & 16.44 & 0.97 & 86.3 & 328.1 & 83.3 & 25.1 & & & 0.44 & 2000.2 & \\
\hline 10 & 18.02 & 14.69 & 1.15 & 62.1 & 395.0 & 117.3 & 39.4 & & 7.9 & 0.32 & 1997.9 & George 1998 \\
\hline 11 & 19.75 & 14.28 & 1.17 & 38.3 & 352.3 & 54.9 & 30.8 & & & 0.34 & 1994.7 & \\
\hline 12 & 19.96 & 14.79 & 1.09 & 62.3 & 367.7 & 87.6 & 21.7 & & & 0.28 & 1991.8 & Klaus 1990 \\
\hline 13 & 22.62 & 14.03 & 1.05 & 60.0 & 353.4 & 119.0 & 28.5 & & & 0.18 & 1988.3 & \\
\hline 14 & 24.43 & 15.36 & 1.02 & 50.8 & 395.5 & 74.0 & 25.9 & & 13.7 & 0.18 & 1982.6 & \\
\hline 15 & 19.56 & 14.71 & 1.06 & 21.4 & 417.3 & 77.7 & 29.6 & & 11.7 & 0.12 & 1977.1 & \\
\hline 16 & 20.34 & 15.01 & 0.91 & 106.2 & 424.4 & 76.1 & 21.9 & 5.0 & & 0.11 & 1968.8 & \\
\hline 17 & 18.24 & 15.11 & 1.05 & 35.7 & 385.6 & 89.5 & 21.9 & & 14.1 & 0.08 & 1959.8 & \\
\hline 18 & 14.39 & 15.79 & 1.20 & 47.4 & 459.1 & 71.6 & 28.1 & & 14.6 & 0.05 & 1947.6 & \\
\hline
\end{tabular}

The sedimentation between $0-33 \mathrm{~cm}$ is similar in relation to calcium and iron; from here until $45 \mathrm{~cm}$, calcium increased, and iron decreased, changing more iron than calcium in the composition. Titanium, similar in nature to iron, diminishes toward the surface. Calcium and strontium, of a similar nature, follow the same trend, changing their relationship in the same sections as the other elements, according to the climatic conditions in the region of the port of Samaná. At $45-47 \mathrm{~cm}$, there was a change in the sedimentation process. At this depth, the values of many elements tend to be constant.

The graphs that relate the TAS and EWP indicate that the Olga and Noel storms did not affect the north coast of the bay, unlike other EWP (Figure (8) due to the trajectory of these phenomena (NOAA, 2019). The most notable changes observed in the sedimentation regime of the C5 core are associated with Hurricane Debby, which occurred in the year 2000: calcium has replaced iron in recent years. Changes in composition also associated with EWP were observed at other depths (Scott et al., 2013). The Yabón River flows into this area, close to the municipality of Sabana de la Mar. After the Yuna, it deposits the most sediments in the bay (Figure 10). Its main basin, formed by karst and volcanic rocks, is in the eastern mountain range. The chemical elements of core $\mathrm{C} 5$ at $0-64 \mathrm{~cm}$ present changes; from $60 \mathrm{~cm}$, the values remain constant, coinciding with the year 1990 and Hurricane Klaus. In the C6 core, the same process occurs with similar geological characteristics as in the C5 core. The sediments come from Los Haitises National Park, formed by karstic rocks, dragged by the Caño Hondo River, and deposited in San Lorenzo Bay. At the mouth of the La Yeguada River in Miches, the C7 core was taken, basically consisting of calcium carbonate. The majority elements vary according to the sedimentation rate, the EWP, and also the minorities of lead and chromium. Core $\mathrm{C} 7$ has the same behavior as cores $\mathrm{C} 5$ and $\mathrm{C} 6$. The changes in the sedimentation rate are related to meteorological eventualities. 

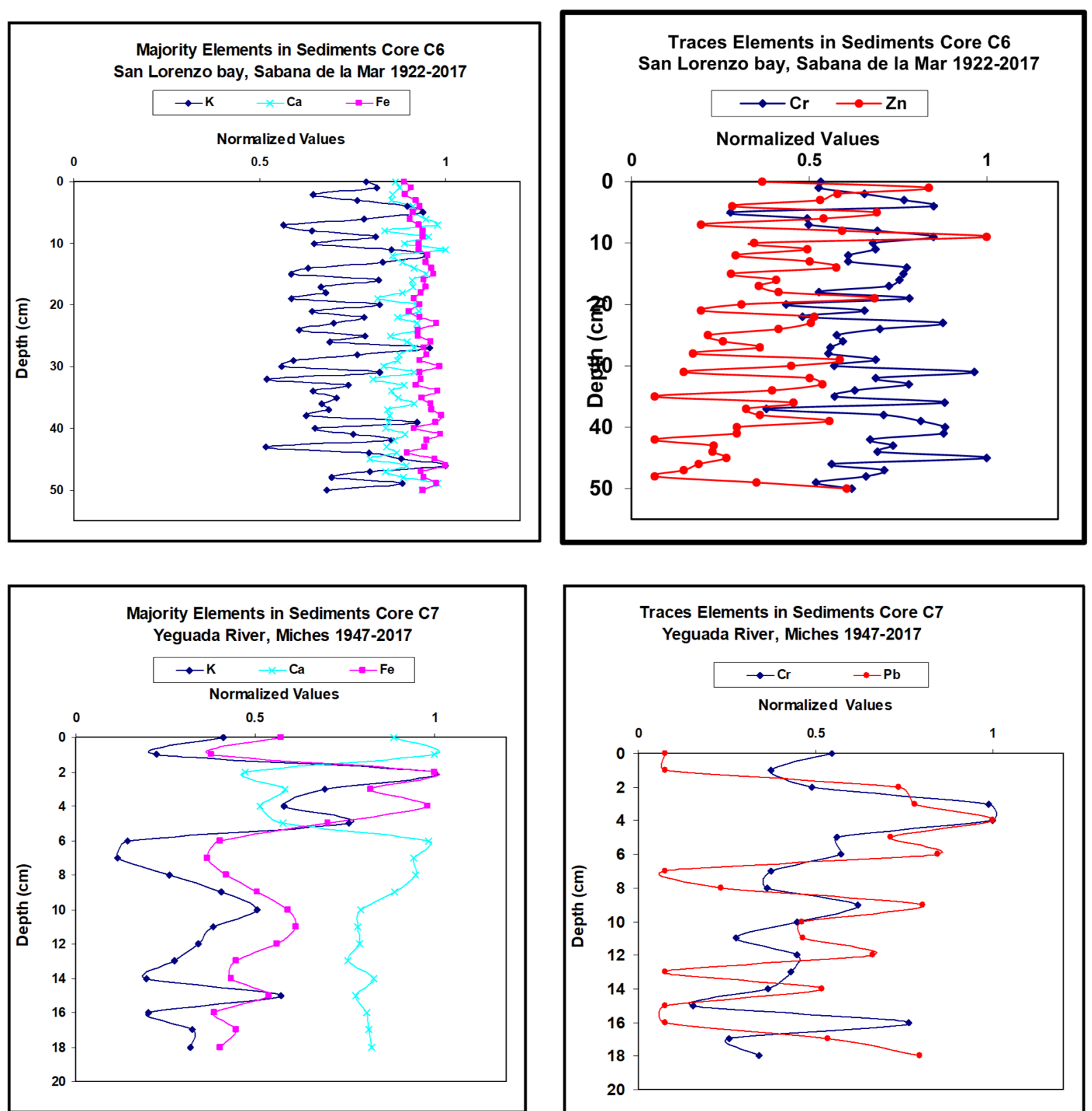

Figure 9. Relationship of the chemical elements with the depth in core C6 (majorities: potassium, calcium, and iron; minorities: chromium and lead). Core C7 (majority: potassium, calcium, and iron; minority: chromium and lead) 


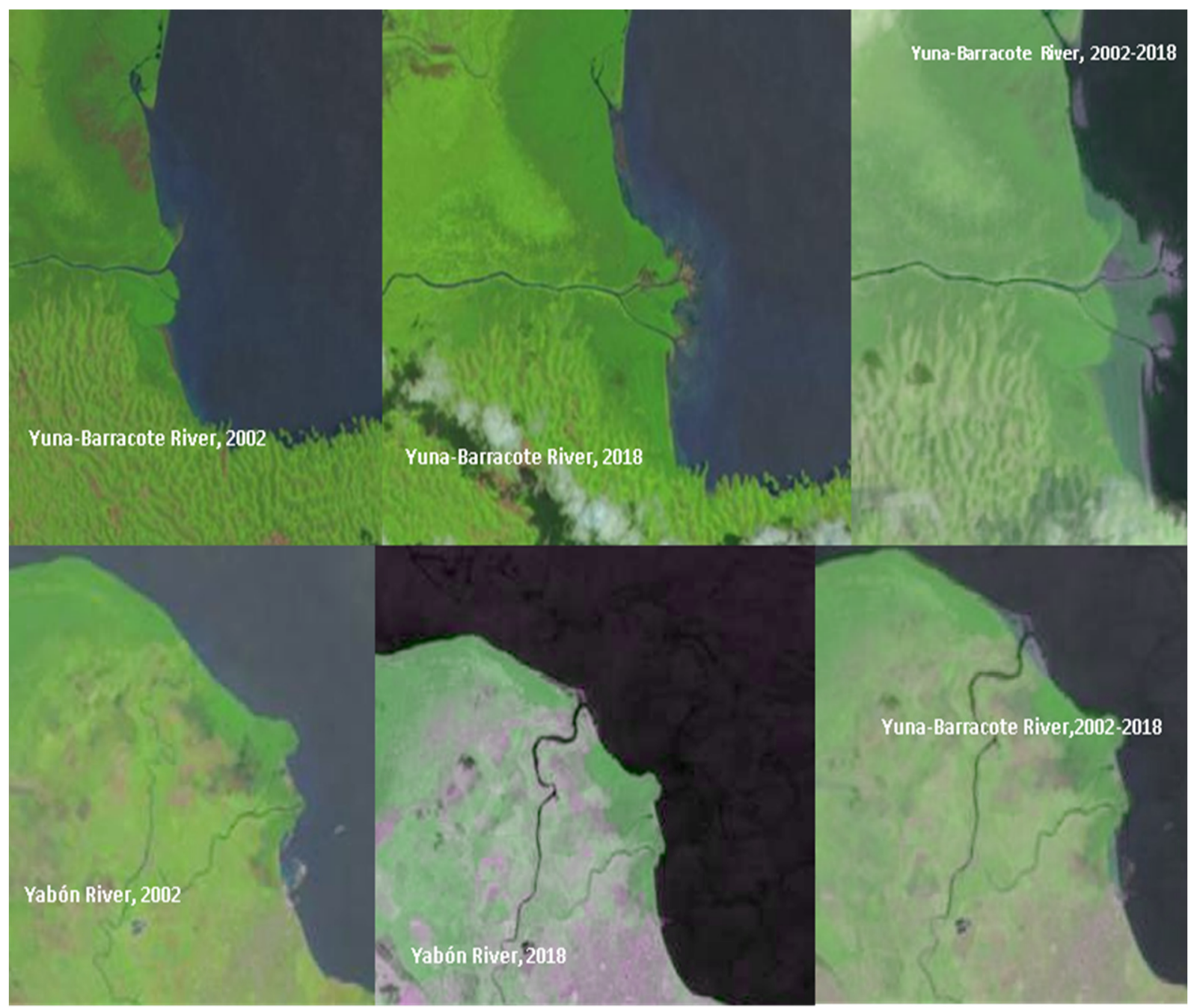

Figure 10. Morphology of the mouths of the Yuna-Barracote and Yabón rivers in 2002 and 2018. Variation of the coastline due to the high rate of sediment accumulation during the period 2002-2018. Source: Landsat 7 Satellite Images (2002) and Landsat 8 (2018)

\section{Discussion}

From the 90's, the EWPs have increased both in frequency and intensity (Goldenberg et al 2001, Villarini et al 2011), because the TAS has also been increased in the Bay of Samaná. There are two other phenomena that have been developed in the DR that are the deforestation of Los Haitises National Park and the mining exploitation in Pueblo Viejo, Cotui.

The sediments, by their origin, contain heavy metals and an important amount of organic matter (from 15 to $24 \%$ ). In the sediments contributed by the Yabón River, the content of organic matter has been decreasing, by which we can assume that this has been very quickly undermining its new channel when it is diverted. In the case of the Caño Hondo River, which flows into the bay of San Lorenzo, the content of organic matter varies between 10 and 15\%. The sediments deposited by the rivers Yuna, Yabón, and La Yeguada have filled the lower parts (beaches) of the coastal area to the south of the bay which, added to the uprisings by the tectonic movements, have caused the land to enter the bay. The sediments deposited by the Yuna River during the period from 2003-2018 have occupied an area of $2.17 \mathrm{~km}^{2}$ in the Bay of Samaná (Figure 9) while those deposited by the rivers Yabón and Yeguada occupy $0.08 \mathrm{~km}^{2}$ and $0.06 \mathrm{~km}^{2}$, respectively.

\section{Conclusions}

In the period from 2007-2016, a sediment deposit that reached $38 \mathrm{~cm}$ and a column of $12 \mathrm{~cm}$ was placed at about $4 \mathrm{~km}$ southeast of the municipality of Sánchez and east of the mouth of the Yuna River. In the bay of San Lorenzo, a column of $22 \mathrm{~cm}(2.2 \mathrm{~km})$ from its mouth was deposited by the Caño Hondo River from Los Haitises. 
In the port of Samaná, a column of more than $28 \mathrm{~cm}$ was deposited in the same period. At the mouths of all the rivers, streams, and gullies of the northern part of the bay, large amounts of sediment were also found. In this period, the storms Noel-Olga, (2007), Ike (2008), Henry (2009), Earl (2010), María-Irene (2011), Dorian (2012), Gabrielle (2013), and Bertha (2016) occurred. In 2017, hurricanes Irma and María hit the entire Samaná Bay, especially the La Yeguada river basin in Miches and deposited a quantity of sediment in the coastal zone.

The behavior of the sediments deposited in Samaná Bay by the rivers Yuna, Yabón, Caño Hondo, and La Yeguada are directly linked to EWP such as troughs and storms, as well as a decrease in these sediments, which can be observed when there are drought episodes (Figure 4). In the first depositions from EWP, the content of organic matter is higher than the next depositions by the trawls, this being recorded in the sediment rates and compositions. Abrupt changes in the rainfall regime produced an equal change in the estuary sedimentation regime, according to the $210 \mathrm{~Pb}$ dating of Sánchez's core (Yuna-Barracote).

\section{Acknowledgment}

To the staff of the Ministry of Higher Education, Science and Technology. Autonomous University of Santo Domingo Dr. Plácido Gómez, MsC. Miledy Alberto, Ing. Alejandro Ozuna, Lic. Domingo Mercedes, Lic. Isabel Ulloa, Maestra Idalia Acevedo, Dr. Carlos Rodríguez, Dr. Carlos Alonso Hernández, MsC Zoraida Zapata, MsC. Miguel Batista, Lic. Marcos Casila, Msc. Nelphy de la Cruz, Yamileza Herrera, Queiroz Portorreal, Droniguiel Jiménez. José Osvaldo Suarez, Juan Pablo González.

\section{References}

Alonso Hernández, C. M., Díaz Asencio, M., Gómez Batista, M., Bolaños Alvares, Y., Muñoz Caravaca, A., \& Morera Gómez, Y. (2016). Radiocronología de sedimentos marinos y su aplicación en la comprensión de los procesos de contaminación ambiental en ecosistemas marinos cubanos. Nucleus, (60), 35-40.

Bionini, W. E., Hargraves, R. B., \& Shagan, R. (1984). The Caribean-South American Plata Boundary and Regional Tectonic. Geological Society of America, Memoir, 162.

Bird, J. M. (1980). Plate tectonics: selected papers from publications of the American Geophysical Union. Plate tectonics: selected papers from publications of the American Geophysical Union, by Bird, John M. Washington, DC: AGU, 1980.. American Geophysical Union.

Brink, U. T., Danforth, W., Polloni, C., Andrews, B., Llanes, P., Smith, S., ... \& Uozumi, T. (2004). New seafloor map of the Puerto Rico Trench helps assess earthquake and tsunami hazards. Eos, Transactions American Geophysical Union, 85(37), 349-354. doi: 10.1029/2004EO370001. issn: 0096-3941.

Delanoy Ramón, A., \& Méndez-Tejeda, R. (2017). Hydrodynamic Study of Lake Enriquillo in Dominican Republic. Journal of Geoscience and Environment Protection, 5, 115-124. doi: 10.4236/gep.2017.55009.

Delanoy, R. A. (1996). Aspectos de sismología dominicana. Ramon A Delanoy.

Díaz de Neira, J. A., \& Hernaiz Huerta, P. P. (2004). Mapa Geológico de la Hoja a escala 1: 50.000 nº 6272-I (Antón Sánchez) y Memoria correspondiente. Dirección General de Minería, Santo Domingo, 125p.

Dolan, J. F., \& Mann, P. (Eds.). (1998). Active strike-slip and collisional tectonics of the northern caribbean plate boundary zone (Vol. 326). Geological Society of America.

Eptisa. (2004). Programa SYSMIN. Informe de la unidad hidrogeológica de la península de Samaná.

Fernández-Domingo, J. I. (2010). Los Tesoros del Mar y su Régimen Jurídico. Biblioteca Iberoamericana de Derecho. Editorial Temis S.A. Bogotá, México D.F., Madrid, Buenos Aires, 2010.

Goldenberg, S. B., Landsea, C. W., Mestas-Nuñez, A. M., \& Gray, W. M. (2001). The recent increase in Atlantic hurricane activity: Causes and implications. Science, 293(5529), 474-479. doi: 10.1126/science.1060040

Hippensteel, S. P., Eastin, M. D., \& Garcia, W. J. (2013). The geological legacy of Hurricane Irene: Implications for the fidelity of the paleo-storm record. GSA Today, 23(12), 4-10.

IAEA. (1989). Isotopes of Noble gases as tracers in environmental studies. Proceeding Consultants Meeeting, Vienna, 29 May-2 June 1989. Agency International.

Lozano, R. L., San Miguel, E. G., \& Bolívar, J. P. (2011). Assessment of the influence of in situ 210Bi in the calculation of in situ 210Po in air aerosols: Implications on residence time calculations using $210 \mathrm{Po} / 210 \mathrm{~Pb}$ activity ratios. Journal of Geophysical Research: Atmospheres, 116(D8).

Mann, P., Burke, K., \& Matumoto, T. (1984). Neotectonics of Hispaniola: Plate motion, sedimentation, and seismicity at a restraining bend. Earth and Planetary Science Letters, 70(2), 311-324. 
Mann, P., Shiroma, J., \& Monechi, S. (1991). Sedimentologic, stratigraphic, and tectonic synthesis of Eocene-Miocene sedimentary basins, Hispaniola and Puerto Rico. Geologic and tectonic development of the North America-Caribbean Plate boundary in Hispaniola, 262, 217.

NCA. (2018). Fourth National Climate Assessment. Retrieved from https://nca2018.globalchange.gov/chapter/20/

NOAA. (2019). Retrieved from https://www.nhc.noaa.gov/data/tcr/index.php?season=2007\&basin=atl

Null, J. (2017). El Niño and La Niña Years and Intensities. Based on Oceanic Niño Index (ONI), CCM. Retrieved from https://ggweather.com/enso/oni.htm

Rodríguez Vegas, E., Gascó Leonarte, C., Schmid, T., Suárez, J. A., \& Rodríguez Rastrero, M. (2014). Estudio preliminar sobre el uso de los radionucleidos ${ }^{137} \mathrm{Cs}$ y ${ }^{210} \mathrm{~Pb}$ y las técnicas de espectrorradiometría como herramientas para determinar el estado de erosión de suelos. Retrieved from http://www.iaea.org/inis/collection/NCLCollectionStore/_Public/45/012/45012641.pdf

Salamanca, M., \& Jara, B. (2003). Distribución y acumulación de plomo (Pb y 210Pb) en sedimentos de los fiordos de la XI Región. Chile. Revista Ciencia y Tecnología del Mar, 26, 2.

Senz, J. G., Monthel, J., de Neira, J. A. D., Huerta, P. P. H., Viruete, J. E., \& Estaun, A. P. (2007). La estructura de la Cordillera Oriental de la República Dominicana. Boletín geológico y minero, 118(2), 293-311.

Villarini, G., Vecchi, G. A., Knutson, T. R., \& Smith, J. A. (2011). Is the recorded increase in short-duration North Atlantic tropical storms spurious?. Journal of Geophysical Research: Atmospheres, 116(D10).

\section{Copyrights}

Copyright for this article is retained by the author(s), with first publication rights granted to the journal.

This is an open-access article distributed under the terms and conditions of the Creative Commons Attribution license (http://creativecommons.org/licenses/by/4.0/). 